\title{
A new limiting procedure for Discontinuous Galerkin methods applied to compressible multiphase flows with shocks and interfaces
}

\author{
Marc T. Henry de Frahan*, Sreenivas Varadan*, Eric Johnsen* \\ Mechanical Engineering Department, University of Michigan, Ann Arbor, MI 48109, USA
}

\begin{abstract}
Although the Discontinuous Galerkin (DG) method has seen widespread use for compressible flow problems in a single fluid with constant material properties, it has yet to be implemented in a consistent fashion for compressible multiphase flows with shocks and interfaces. Specifically, it is challenging to design a scheme that meets the following requirements: conservation, highorder accuracy in smooth regions and non-oscillatory behavior at discontinuities (in particular, material interfaces). Following the interface-capturing approach of Abgrall [1], we model flows of multiple fluid components or phases using a single equation of state with variable material properties; discontinuities in these properties correspond to interfaces. To represent compressible phenomena in solids, liquids, and gases, we present our analysis for equations of state belonging to the Mie-Grüneisen family. Within the DG framework, we propose a conservative, high-order accurate, and non-oscillatory limiting procedure, verified with simple multifluid and multiphase problems. We show analytically that two key elements are required to prevent spurious pressure oscillations at interfaces and maintain conservation: (i) the transport equation(s) describing the material properties must be solved in a non-conservative weak form, and (ii) the suitable variables must be limited (density, momentum, pressure, and appropriate properties entering the equation of state), coupled with a consistent reconstruction of the energy. Further, we introduce a physics-based discontinuity sensor to apply limiting in a solution-adaptive fashion. We verify this approach with one- and two-dimensional problems with shocks and interfaces, including high pressure and density ratios, for fluids obeying different equations of state to illustrate the robustness and versatility of the method. The algorithm is implemented on parallel graphics processing units (GPU) to achieve high speedup.
\end{abstract}

Keywords: Discontinuous Galerkin, Non-conservative equations, Interface capturing, Interface oscillations, High-order limiting, Multiphase and multicomponent flows, Mie-Grüneisen equation

\section{Introduction}

The Discontinuous Galerkin (DG) method is a numerical approach for partial differential equations that combines advantages of the finite element and finite volume (FV) formulations. The solution is discretized locally in each computational cell through an expansion in terms of polynomial basis functions. As such, the DG method is high-order accurate, has a compact stencil that

\footnotetext{
${ }^{*}$ Corresponding author

Email addresses: marchdf@umich.edu (Marc T. Henry de Frahan), ejohnsen@umich.edu (Eric Johnsen)
} 
makes it highly scalable on parallel architectures, and is readily implementable on unstructured grids. The DG approach exhibits superconvergent properties $[2,3]$, at a rate of $2 P+1$ based on cell averages, where $P+1$ is the number of basis functions in each cell. In a series of articles, Cockburn and Shu popularized the Runge-Kutta Discontinuous Galerkin for time-dependent convection-dominated problems $[4,5,6,7,8]$. As with FV methods, Riemann solvers are used to calculate the numerical fluxes between neighboring cells. These solvers provide means to introduce dissipation, so that discontinuities can be treated in a stable fashion. Limiters are further required to damp the oscillations caused by high-order interpolation across a discontinuity. For example, Cockburn and Shu [5] proposed a total variation bounded projection limiter that truncates higherorder Legendre polynomials at detected discontinuities. To prevent excessive dissipation, Biswas et al. [9] developed a hierarchical procedure that limits the Legendre coefficients from the highest to lowest moments. Recently, different high-order limiters have been proposed by Krivodonova [10] and Kuzmin [11]. In particular, hierarchical reconstruction [12, 13] is high-order accurate and conservative; additionally, this compact limiting procedure does not require a characteristic decomposition and is adaptable to unstructured grids.

While the DG method has been used extensively for single-fluid problems [8], the treatment of compressible multicomponent and multiphase flows with interfaces has received little attention. Our present interest lies in interface capturing for the Euler equations, in which interfaces are regularized over a few grid points in analogy to shock capturing. We seek to use methods that conserve the relevant physical quantities (mass, momentum, energy) and are high-order accurate in smooth regions, non-oscillatory at discontinuities, and physically consistent. In the FV formulation, Abgrall [1] showed that spurious pressure oscillations are generated at interfaces between gases of different ratios of specific heats $\gamma$ if the transport equation describing the fluid composition is solved in conservative form. Such errors may lead to negative pressures or trigger unphysical interfacial instabilities. In FV methods, these oscillations can be prevented by solving a nonconservative evolution equation for a specific function of $\gamma[1]$. Multicomponent flows are thus modelled as a medium described by a single equation of state with variable material properties that must be transported in an appropriate form; discontinuities in these properties correspond to interfaces. Saurel and Abgrall [14] and Abgrall and Karni [15] detailed various approaches to solve this problem in the FV context, which can be extended to high-order accuracy $[16,17]$ and finite differences $[18,19]$. Such methods also conserve the total mass, momentum, and energy in the system.

For compressible multiphase problems, the approach of Abgrall [1] can be generalized to more complicated equations of state in the FV context. Transport equations for appropriate materialdependent parameters have been detailed for various equations of state, e.g., stiffened equation [20], Van der Waals [21], Mie-Grüneisen [22]. Such approaches have been used to simulate problems involving shock waves interacting with interfaces separating different fluids [23, 24]. Variations of the physical model, e.g., five- to seven-equations models, have also been explored [25, 26, 27, 28]. These approaches have yet to be extended to the DG framework. Furthermore, based on experience with high-order accurate FV methods [16], we anticipate that limiting may have to be modified to prevent pressure errors.

At this time, a high-order accurate, conservative, non-oscillatory, and consistent treatment of the Euler equations for capturing interfaces between fluids of different composition and/or phase in the DG framework is lacking. Tokareva and Toro [29] and Franquet and Perrier [30] used the DG approach for the Baer-Nunziato system of equations for multiphase flows while Michoski et al. [31] 
solved the compressible Navier-Stokes equations. In these papers, limiting is based on first-order accurate slope limiters proposed by Cockburn and Shu [32]. Franquet and Perrier [33] introduced a maximum-preserving limiter for the color function used to distinguish the different fluids; their limiting procedure truncates the higher-order terms (second order and above) and they do not discuss potential spurious pressure oscillations and conservation issues introduced by the limiting procedure. Wang and Shu [34] used an interface tracking method with a level-set advection equation solved with the RKDG method for Hamilton-Jacobi equations. Gryngarten and Menon [35] extend the Local DG method to the five-equations model [36], in which auxiliary variables are introduced for consistency when solving the Hamilton-Jacobi equations, and a moment limiter [10] is applied to the conserved and primitive variables. Although the amplitude of the pressure oscillations appears to be reduced in this latter article, the authors do not discuss possible conservation issues related to limiting the primitive variables. Several of the above references used solution-adaptive strategies to apply limiting only where needed, i.e., near discontinuities. As stated earlier, these recent DG multiphase flows lack either one or more of the three following properties: conservation of mass, momentum, and energy, non-oscillatory pressure fields at material interfaces, and high-order accuracy. The goal of this work is to fill this gap.

We propose a new approach to limiting in compressible multiphase flows that is conservative, high-order accurate, and that prevents oscillations at interfaces (and shocks). Using a capturing approach for DG, we show that two key elements are necessary to satisfy the three properties listed above: (i) solving transport equations in a non-conservative weak form, and (ii) limiting the suitable variables (density, momentum, pressure and appropriate properties from the equation of state) coupled with a consistent reconstruction of the energy. This procedure is general, and we show how it can be applied to other multiphase models such as the five-equations model and Mie-Grüneisen family of equation of state. This article is organized as follows. In Section 2, we present the physical model, followed by the DG framework and limiting in Section 3. In Section 4, we discuss how spurious pressure oscillations occur in multiphase flows in the DG framework; based on this knowledge, we propose a strategy to avoid these errors through a new limiting procedure in the central part of this study (Section 4.2.2). Finally, we provide numerical verification with various one- and two-dimensional problems in Section 5.

\section{Physical Model}

Neglecting physical diffusion, we consider the Euler equations for compressible flow, written in three dimensions $(i, j=1,2,3)$ :

$$
\begin{aligned}
& \frac{\partial \rho}{\partial t}+\frac{\partial}{\partial x_{j}}\left(\rho u_{j}\right)=0, \\
& \frac{\partial\left(\rho u_{i}\right)}{\partial t}+\frac{\partial}{\partial x_{j}}\left(\rho u_{i} u_{j}+p \delta_{i j}\right)=0, \\
& \frac{\partial E}{\partial t}+\frac{\partial}{\partial x_{j}}\left[u_{j}(E+p)\right]=0,
\end{aligned}
$$

where $\rho$ is the density, $u_{i}$ the velocity, $E=\rho e+\frac{1}{2} \rho u_{i} u_{i}$ the total energy, $e$ the internal energy, $p$ the pressure and $\delta_{i j}$ the Kronecker delta.

The system is closed by an equation of state relating the internal energy to the pressure. We focus on analytical expressions for general compressible phenomena in fluids and solids. For this 
purpose, we consider the Mie-Grüneisen equation of state [37],

$$
p=\left(\rho E-\frac{\rho u_{i} u_{i}}{2}+\frac{p_{\text {ref }}}{\Gamma}-\rho e_{\text {ref }}\right) /\left(\frac{1}{\Gamma}\right),
$$

where $\Gamma=\left.\frac{1}{\rho} \frac{\partial p}{\partial e}\right|_{\rho}$ is the Grüneisen coefficient, $e_{\text {ref }}$ the reference internal energy, and $p_{\text {ref }}$ the reference pressure. A wide range of equations of state belong to the Mie-Grüneisen family, e.g., the Jones-Wilkins-Lee equation for gaseous explosives [38], or the Cochran-Chan equation for solid explosives [39]. For the problems of interest, we assume that the material properties $\left(\Gamma_{\text {ref }}, p_{\text {ref }}, e_{\text {ref }}\right)$ do not vary with density. Setting $e_{\text {ref }}=0$ reduces this equation to the stiffened equation of state for liquids and solids, and setting $p_{\text {ref }}=0$ and $\Gamma=\gamma-1$ further reduces it to the ideal gas law, where $\gamma$ is the specific heats ratio.

To represent multiple components or phases, we follow an approach in which a single equation of state describes all media, but with variable material properties; discontinuities in these properties correspond to interfaces. These properties thus depend on the mass fraction $Y^{(i)}$. Since the mass fraction obeys a mass conservation equation, any function $f\left(Y^{(i)}\right)$ does as well, e.g.,

$$
\frac{\partial}{\partial t}\left(\rho f\left(Y^{(i)}\right)\right)+\frac{\partial}{\partial x_{j}}\left(\rho u_{j} f\left(Y^{(i)}\right)\right)=0, \quad i=1, \ldots, m-1,
$$

where $m$ is the number of different fluids. In this work, we focus on binary systems $(m=2)$. Eq. (3) can be written in advection (non-conservative) form,

$$
\frac{\partial}{\partial t}\left(f\left(Y^{(i)}\right)\right)+u_{j} \frac{\partial}{\partial x_{j}}\left(f\left(Y^{(i)}\right)\right)=0, \quad i=1, \ldots, m-1 .
$$

Although physical diffusion is ignored, mixture regions may occur due to numerical diffusion, thus requiring mixture rules. For instance, the physical relationship between $\gamma$ and $Y^{(i)}$ for a binary gas mixture is

$$
\frac{1}{\gamma-1} \frac{1}{M}=\frac{Y}{\gamma_{1}-1} \frac{1}{M_{1}}+\frac{1-Y}{\gamma_{2}-1} \frac{1}{M_{2}}, \quad \frac{1}{M}=\frac{Y}{M_{1}}+\frac{1-Y}{M_{2}},
$$

where $M$ is the mixture molecular mass and the indices denote fluids one and two. Similar such relationships can be obtained for the other material properties in the Mie-Grüneisen equation of state [24].

\section{Numerical Framework}

\subsection{Discontinuous Galerkin Discretization}

For simplicity, consider the one-dimensional hyperbolic equation

$$
\frac{\partial W}{\partial t}+\frac{\partial F}{\partial x}=0
$$

for the conserved variable $W(x, t)$, where $F(W)$ is the flux, discretized in computational cell $\Omega_{k}=$ $\left\{x \mid x \in\left[x_{k-1 / 2}, x_{k+1 / 2}\right]\right\}$ with uniform grid spacing $\Delta x$. In the DG approach, the solution is expanded in terms of $P+1$ basis functions $\varphi_{n}(x)$ (e.g., Legendre or Lagrange polynomials) in each cell, $W(x, t) \simeq W_{h}(x, t)=\sum_{n=0}^{P} w_{n}(t) \varphi_{n}(x)$, where $W_{h}$ is a projection of $W$ onto a finite-dimensional 
space. Taking the inner product of the conservation law with a basis function over cell $\Omega_{k}$, the weak form of Eq. (6) is obtained

$$
\int_{\Omega_{k}} \varphi_{i} \frac{\partial W}{\partial t} \mathrm{~d} x=\int_{\Omega_{k}} \frac{\mathrm{d} \varphi_{i}}{\mathrm{~d} x} F \mathrm{~d} x-\left[\varphi_{i} \hat{F}\right]_{x_{k-1 / 2}}^{x_{k+1 / 2}}
$$

and the evolution equation for the coefficients $w_{n}(t)$ is

$$
\frac{\Delta x}{2 n+1} \frac{\mathrm{d} w_{n}}{\mathrm{~d} t}=\int_{\Omega_{k}} \frac{\mathrm{d} \varphi_{n}}{\mathrm{~d} x} F \mathrm{~d} x-\left[\left.\hat{F}\right|_{x_{k+1 / 2}}-\left.(-1)^{n} \hat{F}\right|_{x_{k-1 / 2}}\right]
$$

where $\hat{F}$ is an appropriate numerical flux.

The DG discretization is not straightforward for non-conservative equations, such as advection equations. A theoretical framework for determining weak solutions to non-conservative products was proposed by LeFloch [40] and Dal Maso et al. [41]. Parés [42], Castro et al. [43], and Canestrelli et al. [44] developed and implemented these ideas in a FV framework, which was extended to DG by Rhebergen et al. [45] (followed here) and Sollie et al. [46]. The non-conservative equation

$$
\frac{\partial V}{\partial t}+\frac{\partial G}{\partial x}+H \frac{\partial V}{\partial x}=0, \quad G=G(V), \quad H=H(V)
$$

for $V(x, t)$ can be written in weak form

$$
\int_{\Omega} \varphi_{i} \frac{\partial V}{\partial t} \mathrm{~d} x=\int_{\Omega} \frac{\mathrm{d} \varphi_{i}}{\mathrm{~d} x} G \mathrm{~d} x-\int_{\Omega} \varphi_{i} H \frac{\partial V}{\partial x} \mathrm{~d} x-\left[\varphi_{i} \hat{G}-\left\{\left\{\varphi_{i}\right\}\right] H \frac{\partial V}{\partial x}\right]_{x_{k-1 / 2}}^{x_{k+1 / 2}},
$$

where $\{\alpha \alpha\}=\frac{1}{2}\left(\alpha_{L}+\alpha_{R}\right)$ and $L$ and $R$ denote the left and right states at the cell edges. Conservative fluxes are implemented with the usual Riemann solvers. We use the DalMaso-LeFloch-Murat theory [41] to evaluate

$$
\left[H \frac{\partial V}{\partial x}\right]_{x_{k+1 / 2}}=\int_{0}^{1} H\left(\psi\left(\tau ; V^{L}, V^{R}\right)\right) \frac{\partial \psi}{\partial \tau}\left(\tau ; V^{L}, V^{R}\right) \mathrm{d} \tau
$$

where $\psi$ is a path connecting the left and right states. Several approximate Riemann solvers have been developed for non-conservative products. Rhebergen et al. [45] proposed an HLL-like approach [47], which can be extended to Lax-Friedrichs [48], Rusanov [49], and HLLC [50] solvers. Dumbser and Toro [51] extended the Osher solver to non-conservative hyperbolic systems. Finally, Toumi [52] proposed a weak definition of the approximate Riemann solver of Roe [53].

\subsection{Limiting Procedure: Hierarchical Reconstruction}

We use hierarchical reconstruction (HR), developed by Liu et al. [12] and Xu et al. [13] to limit the solution at discontinuities, which preserves high-order accuracy in smooth regions. The idea underlying HR is to recompute the coefficients of the polynomials inside a cell, from the highest degree coefficient to the lowest, e.g., using a MUSCL [54] or WENO [55] approach. The advantage of this hierarchical procedure is that the order of accuracy remains high (although not superconvergent, as demonstrated later). Let the polynomial in cell $k$ be

$$
U_{k}(x)=\sum_{n=0}^{P} c_{k}^{(n)} \frac{x^{n}}{n !} .
$$


The limited coefficient $c_{k}^{(n)}$ is computed by applying a limiter function to candidates of $c_{k}^{(n)}$. To find these candidates, we calculate the $n-1$ derivative of $U_{k}(x)$ in terms of a linear polynomial $L_{k}$ and a higher-order polynomial $R_{k}$ : $\partial^{n-1} U_{k}=L_{k}(x)+R_{k}(x)$. Since the cell averages of $L_{k}$ in the element and the adjacent ones are known, we can form the candidate coefficients for $L_{k}$ and, therefore, candidates for $c_{k}^{(n)}$. In HR, the cell averages in an element $k$ and its neighbors are approximated in the following way:

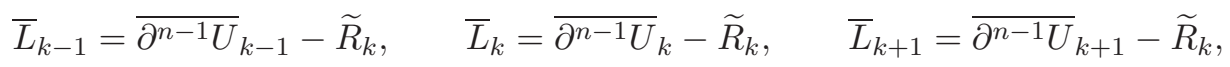

where $\widetilde{R}_{k}(x)$ is the cell average of the remainder polynomial with the limited coefficients and extends into the adjacent elements. The cell averages are then combined, here using a standard MUSCL procedure, to reconstruct the limited first-order coefficient of $L_{k}$ and, equivalently, $c_{k}^{(n)}$. This procedure preserves the cell average of the limited variable. While, in theory, limiting applied to the characteristic variables prevents small oscillations in shock-tube problems [4], this approach is computationally expensive and leads to other problems related to multi-dimensional inconsistencies and unstructured grids [12, 35].

\section{Preventing Spurious Pressure Oscillations at Interfaces}

The DG procedure works well for single-fluid compressible problems (e.g., constant material properties in the equation of state). However, a naive implementation to flows of non-constant material properties generally produces numerical errors. Starting with ideal gases, we show that spurious pressure oscillations may be produced by two mechanisms: (i) an unsuitable form of the transport equation, and (ii) a limiting procedure that does not preserve pressure equilibrium. We then propose an approach that prevents these errors, and extend it to multiphase flows obeying a Mie-Grüneisen equation of state. By considering the advection of an isolated interface between two ideal gases with different values of $\gamma$ moving at a constant velocity $u>0$, we verify our analysis.

\subsection{The Cause for the Oscillations}

\subsubsection{Oscillations due to the Form of the Transport Equation}

We first show that an unsuitable weak form of the transport equation causes spurious pressure oscillations. Let the initial data in two neighboring cells $I-1$ and $I$ be $(\rho, \rho u, E)^{I-1}$ and $(\rho, \rho u, E)^{I}$, respectively, with the interface located between the two cells. For the advection of a material interface, $\rho^{I-1} \neq \rho^{I}$ and $\gamma^{I-1} \neq \gamma^{I}$, but $u^{I-1}=u^{I}$ and $p^{I-1}=p^{I}$. The density, momentum, and energy are $\rho^{I}=\sum_{n=0}^{P} \rho_{n}^{I} \varphi_{n},(\rho u)^{I}=\sum_{n=0}^{P}(\rho u)_{n}^{I} \varphi_{n}, E^{I}=\sum_{n=0}^{P} E_{n}^{I} \varphi_{n}$. Thus, the semi-discrete continuity equation becomes:

$$
\frac{\Delta x}{2 n+1} \frac{\mathrm{d} \rho_{n}^{I}}{\mathrm{~d} t}+\left.\rho u\right|_{x_{I+1 / 2}}-\left.(-1)^{n} \rho u\right|_{x_{I-1 / 2}}-\int_{\Omega_{I}} \rho u \frac{\mathrm{d} \varphi_{n}}{\mathrm{~d} x} \mathrm{~d} x=0 .
$$

We use an upwind flux to approximate its value at $x_{I-1 / 2}\left(\right.$ and $\left.x_{I+1 / 2}\right)$,

$$
\left.\rho u\right|_{x_{I-1 / 2}}=\left.(\rho u)^{I-1}\right|_{x_{I-1 / 2}}=\left.\sum_{m=0}^{P}(\rho u)_{m}^{I-1} \varphi_{m}\right|_{x_{I-1 / 2}}=\sum_{m=0}^{P}(-1)^{m}(\rho u)_{m}^{I-1} .
$$


Since the velocity is positive and constant across the interface between the left and right cells,

$$
\frac{\Delta x}{2 n+1} \frac{\mathrm{d} \rho_{n}^{I}}{\mathrm{~d} t}+u \sum_{m=0}^{P}\left[\rho_{m}^{I}-(-1)^{n+m} \rho_{m}^{I-1}\right]-u \sum_{m=0}^{P} \rho_{m}^{I} \int_{\Omega_{I}} \varphi_{m} \frac{\mathrm{d} \varphi_{n}}{\mathrm{~d} x} \mathrm{~d} x=0
$$

Similarly, for conservation of momentum:

$\frac{\Delta x}{2 n+1} \frac{\mathrm{d}(\rho u)_{n}^{I}}{\mathrm{~d} t}+u^{2} \sum_{m=0}^{P}\left[\rho_{m}^{I}-(-1)^{n+m} \rho_{m}^{I-1}\right]-u^{2} \sum_{m=0}^{P} \rho_{m}^{I} \int_{\Omega_{I}} \varphi_{m} \frac{\mathrm{d} \varphi_{n}}{\mathrm{~d} x} \mathrm{~d} x+p\left[1-(-1)^{n}\right]-p \int_{\Omega_{I}} \frac{\mathrm{d} \varphi_{n}}{\mathrm{~d} x} \mathrm{~d} x=0$,

where the last two terms cancel out. Therefore, the evolution of the $n^{\text {th }}$ transport coefficient is

$$
\frac{\Delta x}{2 n+1} \frac{\mathrm{d}(\rho u)_{n}^{I}}{\mathrm{~d} t}+u^{2} \sum_{m=0}^{P}\left[\rho_{m}^{I}-(-1)^{n+m} \rho_{m}^{I-1}\right]-u^{2} \sum_{m=0}^{P} \rho_{m}^{I} \int_{\Omega_{I}} \varphi_{m} \frac{\mathrm{d} \varphi_{n}}{\mathrm{~d} x} \mathrm{~d} x=0,
$$

such that

$$
\frac{d}{d t}(\rho u)_{n}^{I}=u \frac{d \rho_{n}^{I}}{d t}
$$

Thus, the kinematic interfacial condition (constant velocity) is preserved.

For conservation of energy,

$$
\frac{\Delta x}{2 n+1} \frac{\mathrm{d} E_{n}^{I}}{\mathrm{~d} t}+\left.u(E+p)\right|_{x_{I+1 / 2}}-\left.(-1)^{n} u(E+p)\right|_{x_{I-1 / 2}}-\int_{\Omega_{I}} u(E+p) \frac{\mathrm{d} \varphi_{n}}{\mathrm{~d} x} \mathrm{~d} x=0 .
$$

Noting that the velocity and pressure are uniform initially, we write $E^{I}=p\left(\frac{1}{\gamma-1}\right)^{I}+\frac{1}{2} \rho^{I} u^{2}$. Collecting the kinetic energy terms, which cancel out similar to the momentum equation, and using Eq. (16),

$\frac{\Delta x}{2 n+1} \frac{\mathrm{d}}{\mathrm{d} t}\left\{p\left(\frac{1}{\gamma-1}\right)_{n}^{I}\right\}+u p\left\{\left.\frac{1}{\gamma-1}\right|_{x_{I+1 / 2}}-\left.(-1)^{n} \frac{1}{\gamma-1}\right|_{x_{I-1 / 2}}\right\}-u p \sum_{m=0}^{P} \int_{\Omega_{I}}\left(\frac{1}{\gamma-1}\right)^{I} \frac{\mathrm{d} \varphi_{n}}{\mathrm{~d} x} \mathrm{~d} x=0$.

Noting that, in general, we can express the ratio of specific heats as a function of the mass fraction, $f(Y)$, we expand the temporal derivative to obtain:

$\frac{\Delta x}{2 n+1} \frac{\mathrm{d} p}{\mathrm{~d} t} f(Y)_{n}^{I}+p\left\{\frac{\Delta x}{2 n+1} \frac{\mathrm{d}}{\mathrm{d} t} f(Y)_{n}^{I}+u\left[\left.f(Y)\right|_{x_{I+1 / 2}}-\left.(-1)^{n} f(Y)\right|_{x_{I-1 / 2}}\right]-u \sum_{m=0}^{P} \int_{\Omega_{I}} f(Y)^{I} \frac{\mathrm{d} \varphi_{n}}{\mathrm{~d} x} \mathrm{~d} x\right\}=0$.

The term in braces is a discrete form of a non-conservative evolution equation for $f(Y)$. Recalling Eq. (5),

$$
\frac{1}{\gamma-1}=f(Y)=\frac{1}{\frac{Y}{M_{1}}+\frac{1-Y}{M_{2}}}\left(\frac{Y}{\gamma_{1}-1} \frac{1}{M_{1}}+\frac{1-Y}{\gamma_{2}-1} \frac{1}{M_{2}}\right) .
$$

Thus, because of the nonlinearity of $f$ in $Y$, solving the transport equation for $Y$ in conservative or non-conservative form cannot ensure that the term in the braces in Eq. (22) is identically zero. As a result, the dynamic interfacial condition (pressure equilibrium) is violated: the pressure is no longer uniform at the next time step, or any subsequent, thus leading to spurious pressure oscillations. 


\subsubsection{Oscillations Due to the Limiting Procedure}

Even if the correct form of the transport equation is used, pressure equilibrium may be violated by the limiting procedure, i.e., given a constant pressure at the beginning of a time step, fully conservative limiting may produce pressures that no longer are constant. To show this, let us denote three adjacent computational cells $L: x \in[-3,-1], C: x \in[-1,1]$, and $R: x \in[1,3]$, with

$$
\rho^{L} \neq \rho^{C} \neq \rho^{R}, \quad \gamma^{L} \neq \gamma^{C} \neq \gamma^{R}, \quad u^{L}=u^{C}=u^{R}=u>0, \quad p^{L}=p^{C}=p^{R}=p .
$$

Before limiting, the pressure at the nodal values are equal, e.g., $p(1)=p(-1)$ for $P=1$. To prevent pressure errors, the nodal values after limiting must be the same, i.e., $\widetilde{p}(1)=\widetilde{p}(-1)$, where $\widetilde{p}(x)=\frac{\widetilde{1}}{\gamma-1}\left(\widetilde{E}-\frac{1}{2} \frac{(\widetilde{\rho u})^{2}}{\widetilde{\rho}}\right)$. The following development shows that this condition cannot be met by limiting the conserved variables, specifically the total energy, as traditionally done.

Without loss of generality, consider $P=1$ and HR limiting $[12,13]$. In each cell, let the solution be

$\rho(x)=\rho_{0}+\rho_{1} x, \quad \rho u(x)=(\rho u)_{0}+(\rho u)_{1} x, \quad E(x)=E_{0}+E_{1} x, \quad \frac{1}{\gamma-1}(x)=\left(\frac{1}{\gamma-1}\right)_{0}+\left(\frac{1}{\gamma-1}\right)_{1} x$,

and the limited solution be

$\widetilde{\rho}(x)=\widetilde{\rho}_{0}+\widetilde{\rho}_{1} x, \quad \widetilde{\rho u}(x)=\widetilde{(\rho u)_{0}}+\widetilde{(\rho u)_{1}} x, \quad \widetilde{E}(x)=\widetilde{E}_{0}+\widetilde{E}_{1} x, \quad \widetilde{\frac{1}{\gamma-1}}(x)=\widetilde{\left(\frac{1}{\gamma-1}\right)_{0}}+\widetilde{\left(\frac{1}{\gamma-1}\right)_{1} x}$.

For density, the limiting procedure leads to $\widetilde{\rho}_{0}=\rho_{0}$ and $\widetilde{\rho}_{1}=\operatorname{minmod}\left(\rho_{0}^{C}-\rho_{0}^{L}, \rho_{0}^{R}-\rho_{0}^{C}\right) / 2$.

For momentum, $\widetilde{\rho u}=u \widetilde{\rho}$, such that velocity equilibrium is preserved. Limiting of the conserved variables produces the following slope in energy:

$\widetilde{E}_{1}=\frac{1}{2} \operatorname{minmod}\left(p\left\{\left(\frac{1}{\gamma-1}\right)_{0}^{C}-\left(\frac{1}{\gamma-1}\right)_{0}^{L}\right\}+\frac{u^{2}}{2}\left(\rho_{0}^{C}-\rho_{0}^{L}\right), p\left\{\left(\frac{1}{\gamma-1}\right)_{0}^{R}-\left(\frac{1}{\gamma-1}\right)_{0}^{C}\right\}+\frac{u^{2}}{2}\left(\rho_{0}^{R}-\rho_{0}^{C}\right)\right)$

Since, in general, $\operatorname{minmod}\left(x_{1}+y_{1}, x_{2}+y_{2}\right) \neq \operatorname{minmod}\left(x_{1}, x_{2}\right)+\operatorname{minmod}\left(y_{1}, y_{2}\right)$ for variable $x_{i}$ and $y_{i}$, pressure cannot be factored out of the minmod function.

As a preview of $\S 4.2 .2$, the condition $\widetilde{p}(1)=\widetilde{p}(-1)=p(1)=p(-1)=p$ can be enforced to produce a slope in total energy as follows:

$$
\begin{aligned}
\widetilde{\widetilde{E}}_{1} & =\frac{1}{\left(\frac{1}{\gamma-1}\right)_{0}}\left(E_{0}-\frac{u^{2}}{2} \rho_{0}\right)\left(\widetilde{\frac{1}{\gamma-1}}\right)_{1}+\frac{u^{2}}{2} \widetilde{\rho}_{1} \\
& =p \frac{1}{2} \operatorname{minmod}\left(\left(\frac{1}{\gamma-1}\right)_{0}^{C}-\left(\frac{1}{\gamma-1}\right)_{0}^{L},\left(\frac{1}{\gamma-1}\right)_{0}^{R}-\left(\frac{1}{\gamma-1}\right)_{0}^{C}\right)-\frac{u^{2}}{2} \frac{1}{2} \operatorname{minmod}\left(\rho_{0}^{C}-\rho_{0}^{L}, \rho_{0}^{R}-\rho_{0}^{C}\right) .
\end{aligned}
$$

By construction, such a definition preserves a uniform pressure. Clearly, Eq. (27) does not reduce

to Eq. (28) and therefore does not preserve pressure equilibrium, i.e., $\widetilde{p}=\frac{1}{\gamma-1}\left(\widetilde{E}-\frac{1}{2} \frac{(\widetilde{\rho u})^{2}}{\widetilde{\rho}}\right)=p$. Thus, fully conservative limiting introduces pressure errors in flows with variable $\gamma$. We generalize this approach to arbitrary $P$ and multiphase flows in $\S 4.2 .2$. 


\subsection{Strategy to Prevent Oscillations}

Now that the cause for spurious pressure oscillations has been established, we propose a strategy to avoid these errors. Our approach relies on (i) the transport equation(s) describing the material properties must be solved in a non-conservative weak form, and (ii) the suitable variables must be limited (density, momentum, pressure and appropriate properties entering the equation of state), coupled with a consistent reconstruction of the energy.

\subsubsection{Transport Equations for the Material Properties}

To prevent spurious pressure oscillations, the form of the transport equations for the material properties is crucial. From Eq. (21) in Section 4.1.1, to ensure that the pressure remains constant in time and space (i.e., $d p /\left.d t\right|_{I}=0$ ), the following equation must hold

$$
\frac{\Delta x}{2 n+1} \frac{\mathrm{d}}{\mathrm{d} t}\left(\frac{1}{\gamma-1}\right)_{n}^{I}+u\left\{\left.\frac{1}{\gamma-1}\right|_{x_{I+1 / 2}}-\left.(-1)^{n} \frac{1}{\gamma-1}\right|_{x_{I-1 / 2}}\right\}-u \sum_{m=0}^{P}\left(\frac{1}{\gamma-1}\right)_{m}^{I} \int_{\Omega_{I}} \varphi_{m} \frac{\mathrm{d} \varphi_{n}}{\mathrm{~d} x} \mathrm{~d} x=0,
$$

which is the weak form of

$$
\frac{\partial}{\partial t}\left(\frac{1}{\gamma-1}\right)+u \frac{\partial}{\partial x}\left(\frac{1}{\gamma-1}\right)=0
$$

This result is consistent with the findings of Abgrall [1] for FV methods.

For multiphase flows with the Mie-Grüneisen equation of state (2), a similar analysis shows that the weak form of the following equations must be solved:

$$
\begin{aligned}
& \frac{\partial}{\partial t}\left(\frac{1}{\Gamma}\right)+u \frac{\partial}{\partial x}\left(\frac{1}{\Gamma}\right)=0, \\
& \frac{\partial}{\partial t}\left(\frac{p_{\text {ref }}}{\Gamma}\right)+u \frac{\partial}{\partial x}\left(\frac{p_{\text {ref }}}{\Gamma}\right)=0, \\
& \frac{\partial}{\partial t}\left(\rho e_{\text {ref }}\right)+\frac{\partial}{\partial x}\left(\rho u e_{\text {ref }}\right)=0 .
\end{aligned}
$$

These equations are consistent with those obtained by Shyue [22] for FV methods and could include density-dependent material properties. The difference lies in the fact that the equations must be written in the appropriate weak form for DG, as described in Section 3.1.

\subsubsection{Conservative, High-Order Accurate, and Non-Oscillatory Limiting for DG}

For discontinuous problems, it is necessary to limit the solution to avoid numerical oscillations with high-order methods. Our goal is to ensure that this limiting procedure does not lead to oscillations at interfaces for non-constant $\gamma$ (and other material properties). Let $A(x), B(x), C(x)$, and $U(x)$ be Taylor polynomials inside a cell $\Omega=\{x \mid x \in[-1,1]\}$,

$$
A(x)=\sum_{n=0}^{P} A_{n} \frac{x^{n}}{n !}, \quad B(x)=\sum_{n=0}^{P} B_{n} \frac{x^{n}}{n !}, \quad C(x)=\sum_{n=0}^{P} C_{n} \frac{x^{n}}{n !}, \quad U(x)=\sum_{n=0}^{P} U_{n} \frac{x^{n}}{n !} .
$$

Let $a$ be a constant inside a cell and the limiting operation be denoted by a tilde. Let us assume $A(x), B(x)$, and $U(x)$ are coupled through an algebraic relation $U=f(A, B, C)$, or, equivalently, $C=f^{-1}(A, B, U)$. We seek a limiting procedure for $U$ that is non-oscillatory, i.e., if $C(x)=a$, then $\widetilde{C}(x)=f^{-1}(\widetilde{A}, \widetilde{B}, \widetilde{U})=a$, high-order accurate, and conservative, i.e., $\int \widetilde{U} \mathrm{~d} \Omega=\int U \mathrm{~d} \Omega$. The 
second and third properties can generally be achieved using high-order limiting such as HR $[12,13]$ for single fluids. However, such limiting procedures are nonlinear, which lead to the following difficulties (see proofs thereof in Appendix A): (i) the limited value of a sum is not equal to the sum of limited values, and (ii) limiting a product of functions violates conservation. In other words, $\widetilde{A+B} \neq \widetilde{A}+\widetilde{B}$ and $\int \widetilde{A} \widetilde{B} \mathrm{~d} \Omega \neq \int A B \mathrm{~d} \Omega$. Satisfying these latter two properties is essential to prevent pressure errors due to limiting.

We show how these two difficulties can be overcome to produce a non-oscillatory limiting procedure.

(i) Sum of limited functions. First, let $U(x)=f(A, B, a)=a A+B$. If we limit $U$ as $\widetilde{U}=a \widetilde{A+B}$ and use the limited values of $A$ and $B$ to recover $a$, we obtain:

$$
\widetilde{a}=f^{-1}(\widetilde{A}, \widetilde{B}, \widetilde{U})=\frac{1}{\widetilde{A}}(\widetilde{U}-\widetilde{B})=\frac{1}{\widetilde{A}}(\widetilde{a+B}-\widetilde{B}) .
$$

This expression is generally not equal to $a$ because of the nonlinearity of the limiting procedure. On the other hand, if we compute the limited value of $U$ based on $\widetilde{A}$ and $\widetilde{B}$, i.e., $\widetilde{U}=\widetilde{a A}+\widetilde{B}$, then the constant is recovered:

$$
\widetilde{a}=f^{-1}(\widetilde{A}, \widetilde{B}, \widetilde{U})=\frac{1}{\widetilde{A}}(\widetilde{U}-\widetilde{B})=\frac{1}{\widetilde{A}}(\widetilde{a A}+\widetilde{B}-\widetilde{B})=a .
$$

Hence, limiting the terms in the addition separately ensures that a constant function remains constant after limiting and therefore that this procedure is non-oscillatory. Furthermore, limiting a sum of functions (possibly multiplied by a constant) is conservative:

$$
\int \widetilde{U} \mathrm{~d} \Omega=\int \widetilde{a A} \mathrm{~d} \Omega+\int \widetilde{B} \mathrm{~d} \Omega=a \int A \mathrm{~d} \Omega+\int B \mathrm{~d} \Omega=\int U \mathrm{~d} \Omega .
$$

High-order accuracy is guaranteed as long as the limiting procedure is high-order accurate.

(ii) Product of limited functions. Limiting a product of functions separately violates the conservation property: if $U=f(A, B, C)=A C$ and $\widetilde{U}=\widetilde{A} \widetilde{C}$, then $\int \widetilde{U} \mathrm{~d} \Omega \neq \int U \mathrm{~d} \Omega$. We propose a remedy to this problem. Using the chain rule for the product of $A$ and $C$, the coefficients of $U$ can be expressed in terms of the coefficients of $A$ and $C$

$$
\begin{aligned}
& U_{0}=\left.\frac{\partial^{0} U}{\partial x^{0}}\right|_{0}=\left.\frac{\partial^{0}(A C)}{\partial x^{0}}\right|_{0}=A_{0} C_{0}, \\
& U_{1}=\left.\frac{\partial^{1} U}{\partial x^{1}}\right|_{0}=\left.\frac{\partial^{1}(A C)}{\partial x^{1}}\right|_{0}=A_{1} C_{0}+A_{0} C_{1}, \\
& U_{2}=\left.\frac{\partial^{2} U}{\partial x^{2}}\right|_{0}=\left.\frac{\partial^{2}(A C)}{\partial x^{2}}\right|_{0}=A_{2} C_{0}+2 A_{1} C_{1}+A_{0} C_{2}, \\
& \ldots \\
& U_{n}=\sum_{k=0}^{n}\left(\begin{array}{l}
n \\
k
\end{array}\right) A_{n-k} C_{k},
\end{aligned}
$$

where $\left(\begin{array}{l}n \\ k\end{array}\right)$ is the binomial coefficient. We reconstruct the limited coefficients of $U$ by

$$
\widetilde{U}_{n}=\sum_{k=0}^{n}\left(\begin{array}{l}
n \\
k
\end{array}\right) \widetilde{A}_{n-k} \widetilde{C}_{k}, \quad \text { for } n=0, \ldots, P .
$$


We then impose conservation,

$$
\int \widetilde{U} \mathrm{~d} \Omega=\int U \mathrm{~d} \Omega \quad \Leftrightarrow \quad \sum_{k=0,2,4, \ldots} \frac{2}{(k+1) !}\left(\widetilde{U}_{k}-U_{k}\right)=0 .
$$

These $P+2$ conditions constrain $P+1$ coefficients. We relax these conditions and use

$$
\begin{aligned}
& \widetilde{U}_{n}=\sum_{k=0}^{n}\left(\begin{array}{l}
n \\
k
\end{array}\right) \widetilde{A}_{n-k} \widetilde{C}_{k}, \quad \text { for } n=1, \ldots, P, \\
& \widetilde{U}_{0}=U_{0}-\sum_{k=2,4, \ldots} \frac{1}{(k+1) !}\left(\widetilde{U}_{k}-U_{k}\right) .
\end{aligned}
$$

If $C=a$, then $C_{0}=a$ and $C_{k}=0$ for $k>0$, so that this procedure reduces to $\widetilde{U}=a \widetilde{A}$, thus verifying the non-oscillatory condition. This procedure naturally preserves high-order accuracy.

In conclusion, the present limiting procedure is conservative and non-oscillatory, and preserves the high-order moments of the solution. Additionally, this procedure requires approximately the same number of operations as fully conservative limiting. This discussion is general and applies to different limiting approaches beyond HR.

\subsubsection{Application to Multiphase Flows}

The derivations above can be directly applied to multiphase flows. It is immediately clear that limiting the total energy in the multiphase Euler equations results in oscillatory pressure fields. It is therefore necessary to apply our modified limiting procedure by reconstructing coefficients of the total energy through

$$
\begin{aligned}
& \widetilde{E}_{n}=\widetilde{\rho e}_{n}+\widetilde{K}_{n}, \quad \text { for } n=1, \ldots, P \\
& \widetilde{E}_{0}=E_{0}-\sum_{k=2,4, \ldots} \frac{1}{(k+1) !}\left(\widetilde{E}_{k}-E_{k}\right),
\end{aligned}
$$

where the limited kinetic energy $\widetilde{K}$ is reconstructed from the limited density and momentum polynomials. Limiting the density and momentum, instead of $\rho$ and $u$, ensures that the method is conservative with respect to those variables. The limited internal energy polynomial, $\widetilde{\rho}$, is reconstructed to ensure that the pressure remains non-oscillatory. For the different equations of state, this implies:

- Ideal gases: we limit $p$ and $\frac{1}{\gamma-1}$, and reconstruct the internal energy as

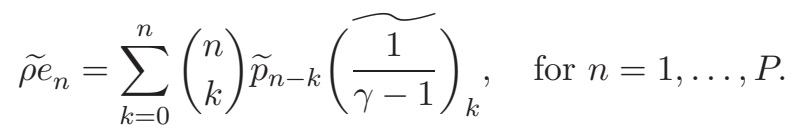

- Stiffened equation of state with $\rho e=\frac{p+\gamma B}{\gamma-1}$, where $\gamma$ and $B$ are constant: we limit $p, \frac{1}{\gamma-1}$ and $\frac{\gamma B}{\gamma-1}$, and reconstruct the internal energy as

$$
\widetilde{\rho e}_{n}=\sum_{k=0}^{n}\left(\begin{array}{l}
n \\
k
\end{array}\right) \widetilde{p}_{n-k}\left(\widetilde{\left.\frac{1}{\gamma-1}\right)_{k}}+\widetilde{(\widetilde{\gamma B}}\right)_{n}, \quad \text { for } n=1, \ldots, P .
$$


- Mie-Grüneisen equation: we limit $p, \frac{1}{\Gamma}, \frac{p_{\text {ref }}}{\Gamma}$ and $\rho e_{\text {ref }}$, and reconstruct the internal energy as

$$
\widetilde{\rho e_{n}}=\sum_{k=0}^{n}\left(\begin{array}{l}
n \\
k
\end{array}\right) \widetilde{p}_{n-k} \widetilde{\left(\frac{1}{\Gamma}\right)_{k}}-\widetilde{\left(\frac{p_{\text {ref }}}{\Gamma}\right)_{n}}+\widetilde{\left(\rho e_{\text {ref }}\right)_{n}}, \quad \text { for } n=1, \ldots, P .
$$

By contrast to other limiting approaches, e.g., [35], our proposed reconstruction, while relying on limiting pressure, is not a straightforward application of limiting the primitive variables, as this would lead to conservation errors (see Section 5.2). Our limiting approach is readily extended to other multiphase models such as the five-equations model (see Appendix B).

\subsection{Verification}

We now verify that the proposed approach is oscillation-free, high-order accurate, and conservative. For simplicity, we consider gases; similar tests can readily be performed for the other equations of state under consideration. We define the $L_{\infty}$ error in the cell average of a quantity $U$ as

$$
L_{\infty}=\max _{i=1 \ldots N_{E}}\left|\frac{1}{\Delta x} \int_{\Omega_{i}} U_{\text {exact }} \mathrm{d} x-\frac{1}{\Delta x} \int_{\Omega_{i}} U_{\text {numerical }} \mathrm{d} x\right|,
$$

where $N_{E}$ is the number of cells in the mesh and the integrals are evaluated with a Gaussian quadrature. In our comparisons, we consider three approaches:

A. Conservative transport equation for $\rho Y$ with the physical relationship between $Y$ and $\gamma(23)$ assuming $M=M_{1}=M_{2}$ as in [20], and limiting of the conserved variables $(\rho, \rho u, E, \rho Y)$, which we call the "fully conservative approach,"

B. Non-conservative transport equation for $1 /(\gamma-1)$ and limiting of the conserved variables $(\rho, \rho u, E)$ and $1 /(\gamma-1)$,

C. Non-conservative transport equation for $1 /(\gamma-1)$ and limiting of the variables $(\rho, \rho u, p, 1 /(\gamma-$ 1)), which we call "our approach."

\subsubsection{Verification of the Non-Oscillatory Property: Sharp Interface Advection}

We consider the advection of sharp isolated material interfaces to show that both an appropriate form of the transport equation and our modified limiting are necessary to prevent pressure oscillations. Two interfaces, characterized by discontinuities in $\rho$ and $\gamma$, are advected at a constant velocity $u=1$ and under constant pressure in the periodic domain $x \in[-1,1]$. The initial conditions are

$$
(\rho, u, p, \gamma)= \begin{cases}(1,1,1,1.4), & \text { for } x \in[-0.5,0.5] \\ (0.125,1,1,1.6), & \text { otherwise }\end{cases}
$$

Because of the sharp interfaces, limiting is required. The exact solution for this problem is a translation of the initial profiles, with velocity and pressure remaining constant throughout.

Fig. 1 shows the pressure field at $t=2$ (after one period) for the three approaches $\mathrm{A}, \mathrm{B}$, and C. The $L_{\infty}$ error in the cell averages for pressure is $\mathcal{O}\left(10^{-2}\right)$ with the fully conservative approach (A); it is smaller for approach $\mathrm{B}\left(\mathcal{O}\left(10^{-3}\right)\right)$. For the proposed approach $(\mathrm{C})$, it is essentially negligible $\left(\mathcal{O}\left(10^{-11}\right)\right)$. The pointwise errors are approximately two orders of magnitude larger. The errors for the first two approaches are observed to propagate away from the interface and affect the solution in the entire domain. The amplitude of these oscillations may grow physically upon interaction with other flow features. While these oscillations are small in this test case, we show 


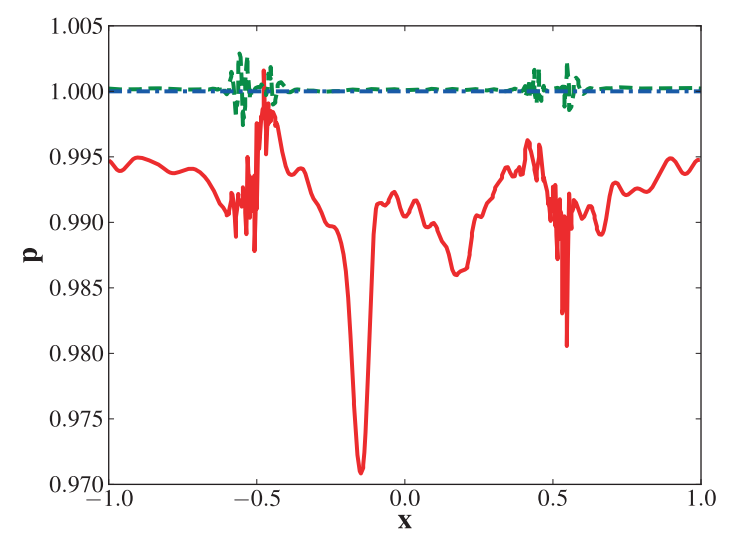

Figure 1: Pressure profile at $t=2$ for the advection of a sharp material interface $(P=2, \Delta x=$ 1/128). Solid red: conservative transport equation and limiting of the conserved variables (fully conservative approach). Dashed green: non-conservative equation and limiting of the conserved variables. Dash-dotted blue: non-conservative equation and modified limiting (our approach).

in later validation tests that they propagate in the flow field, interact with other flow features, and cause the simulations to fail (Sections 5.3 and 5.4). It is clear that the errors arise for two reasons: the form of the transport equation for the material properties and the limiting procedure. The non-conservative formulation coupled with our modified limiting (our approach C) presents an oscillation-free pressure.

\subsubsection{High-Order Property: Smooth Variation in $\gamma$}

We consider the advection of a smooth variation in $\gamma$ and density to ensure that the proposed approach does not affect the convergence rate. This distribution moves at a constant velocity $u=1$ and under constant pressure in the periodic domain $x \in[-1,1]$. The initial conditions are

$$
(\rho, u, p, \gamma)=(1+0.2 \sin (4 \pi x), 1,1,1.4+0.2 \sin (\pi x)) .
$$

Since this problem is smooth, a stable solution can be achieved without limiting. The exact solution for this problem is a translation of the initial profiles, with velocity and pressure remaining constant throughout.

We first consider the solution to this problem with no limiting, to ensure that the non-conservative form of the transport equation produces the expected convergence rate, independently of limiting. Fig. 2 shows the $L_{\infty}$ error in cell averages of $1 /(\gamma-1)$ and pressure after one period for the conservative and non-conservative transport equations (e.g., comparing approaches $\mathrm{A}$ and $\mathrm{B} / \mathrm{C}$ with no limiting). For pressure, errors are produced with the conservative approach, and decrease at the expected rate of convergence for DG $(2 P+1)$; a sufficiently fine resolution is required to achieve this convergence rate. With the non-conservative approach, errors lie at the round-off level for all grids. For $1 /(\gamma-1)$, the convergence rate is $2 P+1$ in both cases. The error is slightly larger for the conservative approach due to the pressure oscillations that affect all fields.

We now consider the solution to this problem with limiting, to evaluate its effect on the convergence rate. In Fig. 3, we compare the three approaches A, B and C. Overall, limiting reduces 


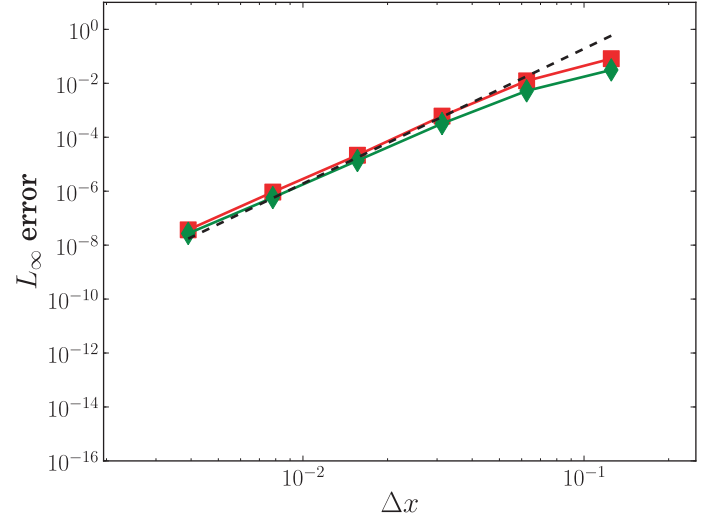

(a) $\frac{1}{\gamma-1}$.

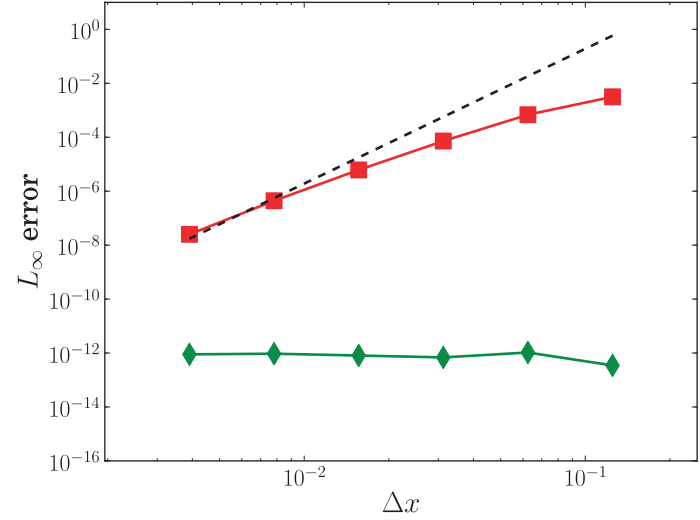

(b) Pressure.

Figure 2: $L_{\infty}$ cell-average error vs. $\Delta x$ for the advection of a smooth distribution in $\gamma$ with no limiting $(P=2)$. Red squares: conservative transport equation. Green diamonds: non-conservative equation. Dashed line: $2 P+1$ slope.

the convergence rate to $P+1$ at best, as expected. Again, approach A (conservative equation and limiting) produces the largest pressure errors. In this case, the pressure oscillations affect all fields and reduce the convergence rate in $1 /(\gamma-1)$; the errors decrease at a rate lower than $P+1$. The non-conservative form of the equations with limiting of the conserved variables (approach B) exhibits minor errors in $\gamma$; the pressure errors do not decrease at rate $P+1$ until a sufficiently fine grid is used. Our approach C produces round-off errors in pressure, as expected.

These problems illustrate two points. First, pressure oscillations are prevented only by both solving a suitable form of the transport equation and limiting the appropriate variables; the fully conservative approach A produces the largest errors. Second, even if no pressure oscillations are produced, limiting significantly reduces the convergence rate in smooth regions $(P+1$ instead of $2 P+1)$. To prevent this reduction in accuracy in flows with smooth and discontinuous features, a solution-adaptive approach is presented in Section 4.4, in which a sensor discriminates between discontinuities in $\gamma$, where our limiting approach should applied, and smooth regions, in which no limiting should be used, e.g., as in [19] for finite differences.

\subsubsection{Conservation Property}

Our proposed limiting procedure is conservative by construction, in that it preserves the cell averages of the relevant conserved variables and the telescoping property of the fluxes. Although we do not display results for conciseness, we verified that energy is conserved using the two above verification problems. In both verification cases, the error in energy lies at the round-off level for all approaches.

\subsection{Solution-Adaptive Approach: Discontinuity Sensors}

The numerical treatment of discontinuities (introducing numerical dissipation), even with highorder accurate methods, lies in direct contradiction with that of smooth broadband motions (preventing numerical dissipation). For such problems, applying limiting only where needed and using 


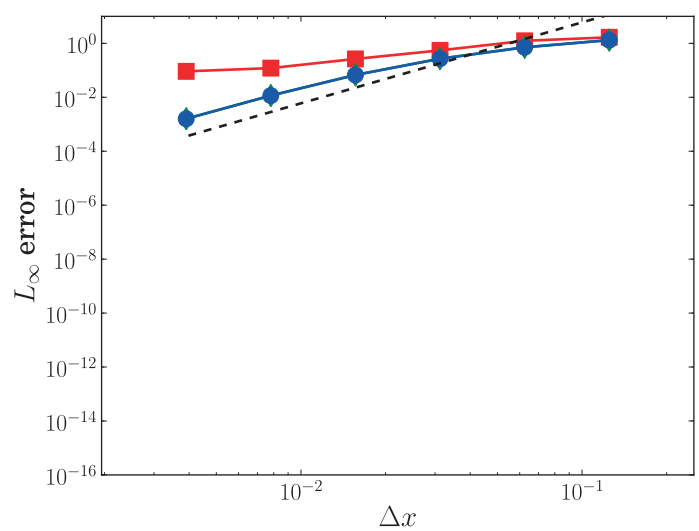

(a) $\frac{1}{\gamma-1}$.

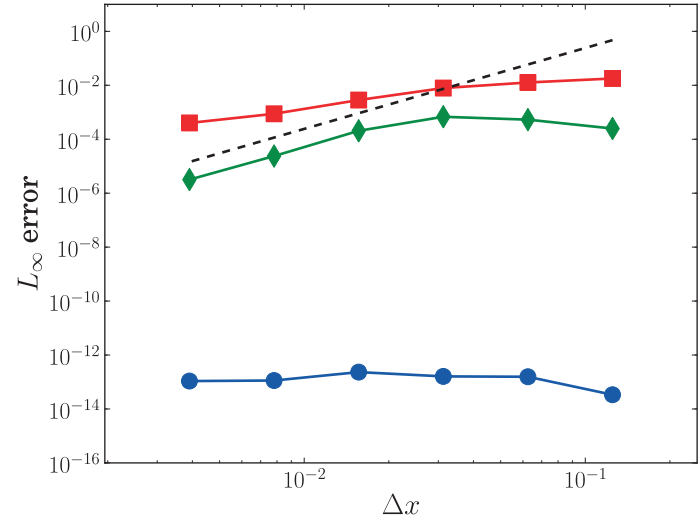

(b) Pressure.

Figure 3: $L_{\infty}$ cell-average error vs. $\Delta x$ for the advection of a smooth distribution in $\gamma$ with limiting $(P=2)$. Red squares: conservative transport equation and limiting of the conserved variables (fully conservative approach A). Green diamonds: non-conservative equation and limiting of the conserved variables (approach B). Blue circles: non-conservative equation and modified limiting (our approach $\mathrm{C}$ ). Dashed line: $P+1$ slope.

non-dissipative methods elsewhere constitutes a more accurate and efficient approach [56]. To discriminate between smooth and discontinuous regions, a discontinuity sensor is required. The overall accuracy and efficiency strongly depends on the discontinuity sensor. However, designing such sensors that are independent of the numerical scheme is challenging. Thus, there is a need for physics-based discontinuity sensors. Another difficulty with hybrid finite difference/volume methods is that the stencil size increases with order of accuracy. As a result, transition regions between the capturing and central schemes exist [57]. Because of its compactness, the DG approach allows for the use of high order immediately next to cells that contain discontinuities.

A characteristics-based sensor inspired by the physics is developed for the Euler equations. In a first sweep, the sensor first identifies cells affected by material/contact discontinuities. Then, shock waves are detected. A different approach is used for each feature. First, we consider interfaces/contacts. At each element interface, let $L$ and $R$ denote the cell-average value of the corresponding variable on the left and right cell, respectively. The discrete characteristic variables for the Euler equations are

$$
\Delta \alpha=\mathcal{R}^{-1} \Delta W, \quad \Delta W=W_{R}-W_{L}=\sum_{i=1}^{3} \widehat{\Delta \alpha_{i}} \widehat{\mathcal{R}}^{(i)},
$$

where $\mathcal{R}$ is the matrix of right eigenvectors, $W$ the vector of conserved variables, $\alpha$ the vector of characteristic variables, and the hat values denote average values, e.g., Roe's [53]. Specifically, the strength of a contact discontinuity propagating the density jump $\Delta \rho$ is

$$
\Delta \widehat{\alpha_{2}}=\frac{\Delta \rho \hat{c}^{2}-\Delta p}{\hat{c}^{2}}
$$


where $c$ is the speed of sound. This quantity is used as a sensor for contact discontinuities, with the following normalization:

$$
\xi=\frac{\left|\Delta \alpha_{2}\right|}{\rho_{L}+\rho_{R}}, \quad \Xi=\frac{2 \xi}{(1+\xi)^{2}} .
$$

Similarly, we construct a sensor for the multifluid Euler equations based $\gamma$,

$$
\zeta=\frac{\left|\gamma_{R}-\gamma_{L}\right|}{\gamma_{L}+\gamma_{R}}, \quad Z=\frac{2 \zeta}{(1+\zeta)^{2}} .
$$

If $\Xi$ or $Z$ is greater than 0.01 , limiting is used in the cells that share that interface.

To detect shocks, we use the sensor developed by Lombardini [58]. At each cell edge, we test the Lax entropy condition, which is satisfied by a physical shock wave:

$$
u_{L}-c_{L}>\hat{u}-\hat{c}>u_{R}-c_{R} .
$$

If this condition is satisfied at the interface, the following pressure sensor is used:

$$
\phi=\frac{\left|p_{R}-p_{L}\right|}{p_{L}+p_{R}}, \quad \Phi=\frac{2 \phi}{(1+\phi)^{2}} .
$$

with a threshold of 0.001 .

These sensors lead to robust detection of shocks and discontinuities and are straightforward to implement in multiple dimensions. Though the thresholds are chosen to be widely applicable (to all problems under consideration), it is likely that an optimal value is problem-dependent.

\subsection{Algorithm}

Our proposed algorithm for interface capturing of compressible multiphase flows using DG can be summarized as follows:

1. Given the coefficients $w_{n}(t)$ at time $t$,

2. Identify the cells that require limiting with our proposed solution-adaptive approach,

3. In the detected cells, limit the flow variables $(\rho, \rho u, p)$ and the appropriate material properties, e.g., $1 /(\gamma-1)$ for ideal gases, $1 /(\gamma-1)$ and $\gamma B /(\gamma-1)$ for the stiffened equation of state, or $1 / \Gamma, p_{\text {ref }} / \Gamma$ and $\rho e_{\text {ref }}$ for the Mie-Grüneisen equation,

4. Reconstruct the internal energy coefficients according to our non-oscillatory procedure following Eq. (41), (42) or (43),

5. Use the DG discretization to march the Euler equations (1) forward in time, and the nonconservative transport equations for the material properties, i.e., Eqs. (31).

\section{Numerical Tests}

We use one- and two-dimensional test problems involving shock waves and interfaces for different equations of state to illustrate the robustness and versatility of our method. For all problems, the standard explicit fourth-order Runge-Kutta method is used, with a Courant number of 0.5. All the numerical tests were performed with the Roe solver and a Lagrange basis. Due to discontinuities, HR with discontinuity sensors is used in all problems. We implemented a highly parallel version of the code on graphics processing units (GPUs), which allows for simulations approximately two orders of 


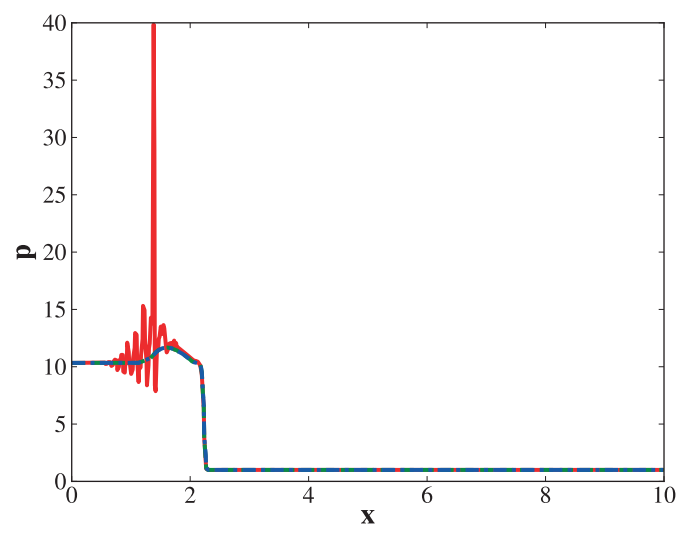

Figure 4: Pressure profile at $t=0.31$ for the multifluid Shu-Osher problem $(P=2, \Delta x=1 / 30)$. Solid red: conservative transport equation and limiting of the conserved variables (fully conservative approach A). Dashed green: conservative equation and limiting of the conserved variables (approach B). Dash-dotted blue: non-conservative equation and modified limiting (our approach C).

magnitude faster than on a single CPU. Multi-GPU parallelism using the Message Passing Interface is implemented to achieve high speedup for large problems. The two-dimensional simulations were performed using GPUs on the Flux cluster at the Center for Advanced Computing at the University of Michigan. Gmsh is used for the mesh generation and post-processing visualization [59]. Our code has been used previously to simulate the multilayered Richtmyer-Meshkov and Rayleigh-Taylor instabilities [60] and experiments of blast-wave-driven shear flow in high-energy-density regimes [61].

\subsection{Multifluid Shu-Osher Problem}

We consider the Shu-Osher problem [62], extended to include two ideal gases of different $\gamma$ [19]. A Mach $M_{s}=3$ shock interacts with a sharp material interface preceding a smooth variation in density and $\gamma$. The initial conditions are

$$
\left(\rho, u, p, \frac{1}{\gamma-1}\right)= \begin{cases}(3.857143,2.629369,10.3333,2.5), & \text { for } x \leq 1, \\ (1+0.2 \sin (5(x-5)), 0,1,1.33+0.2 \sin (5(x-5))), & \text { otherwise. }\end{cases}
$$

In Fig. 4, we compare the pressure profile for approaches $\mathrm{A}, \mathrm{B}$, and $\mathrm{C}$ at early time. The fully conservative approach A leads to large pressure oscillations and the simulation fails at the illustrated time. The pressure oscillations with approach B are small enough to not significantly impact the solution in this problem. Our approach $\mathrm{C}$ does not exhibit any pressure oscillations and adequately represents the solution at time 1.8 (Fig. 5). As with finite difference methods for similar resolutions, the entropy waves are damped as they propagate away from the shock [56].

\subsection{Strong Shock Impacting on an Interface}

We consider a strong shock (Mach 8.96) interacting with a moving gas-gas interface [63]. We denote the post-shock region with index $4(-1<x<-0.8)$, the pre-shock region with the first gas 


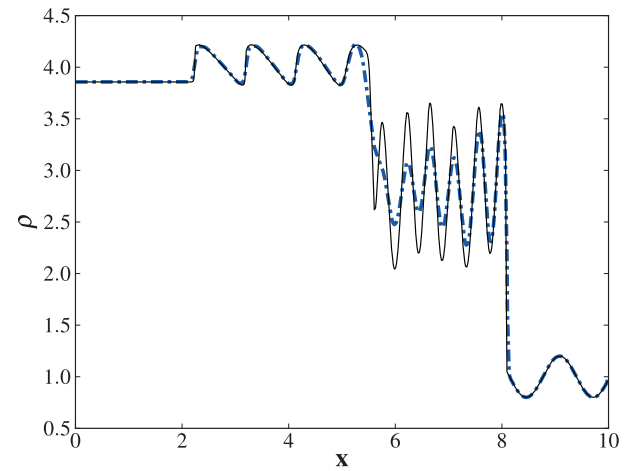

(a) Density.

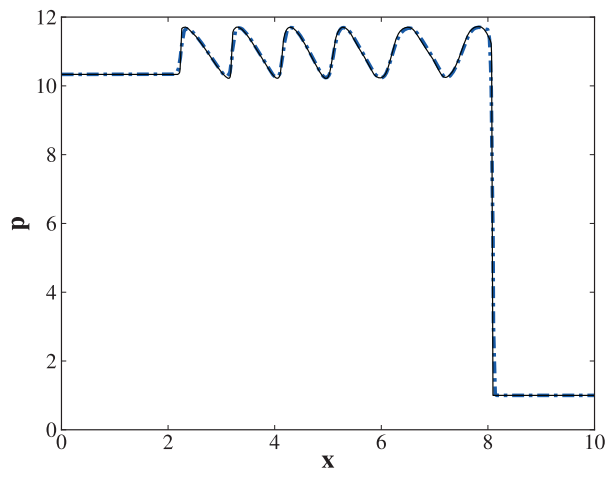

(c) Pressure.

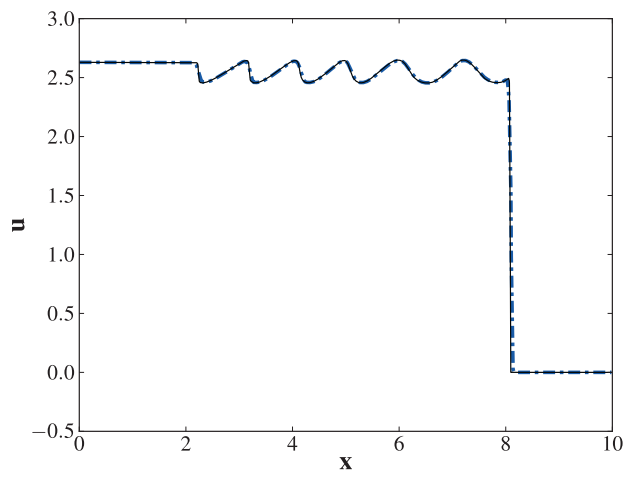

(b) Velocity.

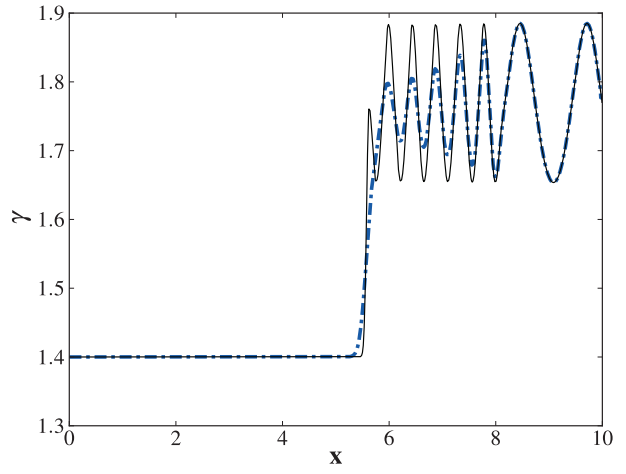

(d) Ratio of specific heats.

Figure 5: Profiles at $t=1.8$ for the multifluid Shu-Osher problem using our approach $\mathrm{C}(P=2)$. Solid black: reference solution (1280 cells). Dash-dotted blue: (300 cells).

by $02(-0.8<x<-0.2)$, and the pre-shock region with the second gas by $01(-0.2<x<1)$. The initial conditions for this problem are:

$\rho=\left\{\begin{array}{l}\rho_{4}=\rho_{02} \frac{\left(\gamma_{02}+1\right) M_{s}^{2}}{\left(\gamma_{02}-1\right) M_{s}^{2}+2}, \\ \rho_{02}=0.1, \\ \rho_{01}=1.0,\end{array} \quad u=\left\{\begin{array}{l}u_{4}=\frac{c_{02}}{M_{s}} \frac{2\left(M_{s}^{2}-1\right)}{\gamma_{02}+1}+u_{c}, \\ u_{02}=u_{c}, \\ u_{01}=u_{c},\end{array} \quad p=\left\{\begin{array}{l}p_{4}=r p_{0}, \\ p_{02}=p_{0}, \\ p_{01}=p_{0},\end{array} \quad \gamma=\left\{\begin{array}{l}\gamma_{4}=\gamma_{02}, \\ \gamma_{02}=\frac{5}{3}, \\ \gamma_{01}=1.4,\end{array}\right.\right.\right.\right.$

with $r=\frac{p_{4}}{p_{0}}=100$ and $p_{0}=1$ such that $M_{s}=\sqrt{\frac{\gamma_{02}+1}{2 \gamma_{02}} r+\frac{\gamma_{02}-1}{2 \gamma_{02}}}=8.96$. We add a background velocity, $u_{c}=-2$, so that the interesting flow features remain near the middle of the domain. As a result, the interface dissipates slightly as it moves to the left before interacting with the shock. Fig. 6 shows the density, velocity, pressure, and $\gamma$ profiles at $t=0.04$ for $P=2$. Limiting the total energy (approach B) leads to significant oscillations in pressure (5\% overshoot near the contact) and velocity. Approach A produce $11 \%$ overshoots in pressure (data not shown). These errors are avoided with our proposed approach $(\mathrm{C})$. The small bumps in $u$ and $p$ around $x=-0.6$ are due to 


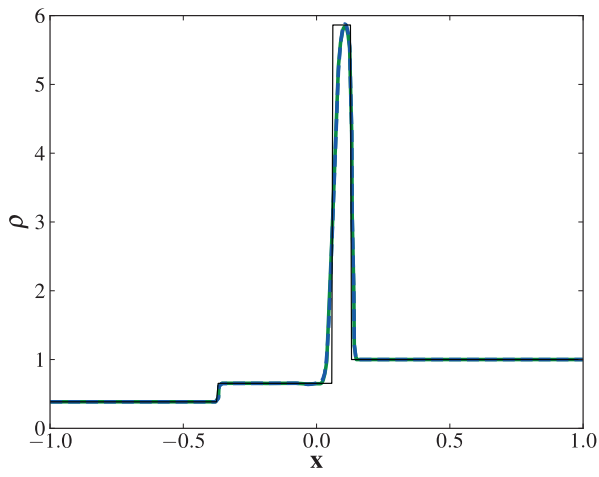

(a) Density.

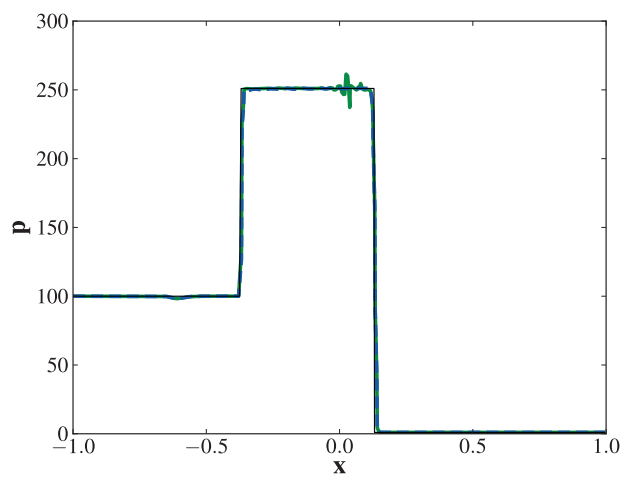

(c) Pressure.

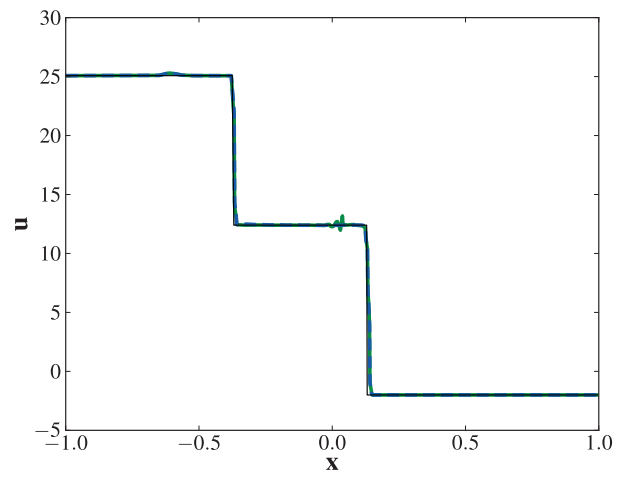

(b) Velocity.

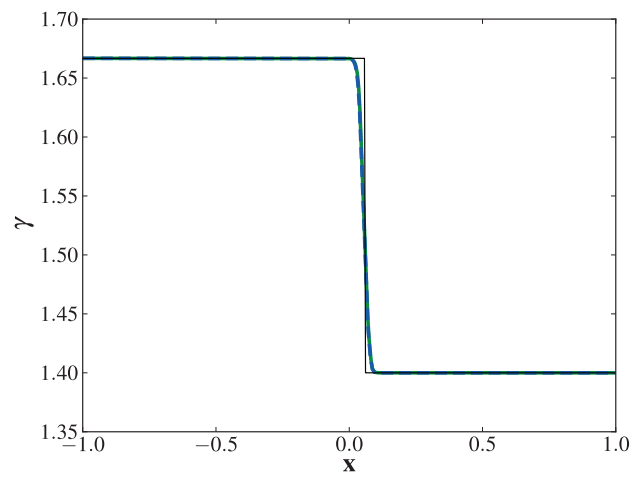

(d) Ratio of specific heats.

Figure 6: Profiles at $t=0.04$ for the strong shock-interface interaction $(P=2, \Delta x=1 / 128)$ using the non-conservative equation for $1 /(\gamma-1)$. Solid green: limiting of the conserved variables (approach B). Dashed blue: modified limiting (our approach C). Solid black: exact solution.

shock startup errors $[64,65]$.

This particular case illustrates the importance of the conservative nature of our approach. If one directly limits the primitive variables $(\rho, u, p)$ as suggested in [35], energy conservation errors are $\mathcal{O}\left(10^{-1}\right)$, compared to $\mathcal{O}\left(10^{-14}\right)$ for our proposed approach. Such errors lead to incorrect shock speeds and interface positions.

\subsection{Richtmyer-Meshkov Instability}

We use the single-mode Richtmyer-Meshkov Instability (RMI) experiments from [66] for validation. A shock $\left(M_{s}=1.21\right)$ initialized in air $\left(\rho_{\text {air }}=1.351 \mathrm{~kg} / \mathrm{m}^{3}, \gamma_{\text {air }}=1.276, M_{\text {air }}=\right.$ $34.76 \mathrm{~kg} / \mathrm{kmol}$ ) and moving downwards impinges a perturbed interface between the air and the denser $\mathrm{SF}_{6}\left(\rho_{S F_{6}}=5.494 \mathrm{~kg} / \mathrm{m}^{3}, \gamma_{S F_{6}}=1.093, M_{S F_{6}}=146.05 \mathrm{~kg} / \mathrm{kmol}\right)$, thereby initiating the RMI growth through baroclinic vorticity deposition. The initial perturbation amplitude and wavelength are $A_{0}=0.183 \mathrm{~cm}$ and $\lambda_{0}=5.933 \mathrm{~cm}$. The diffuse interface is initialized in a thermodynamically 

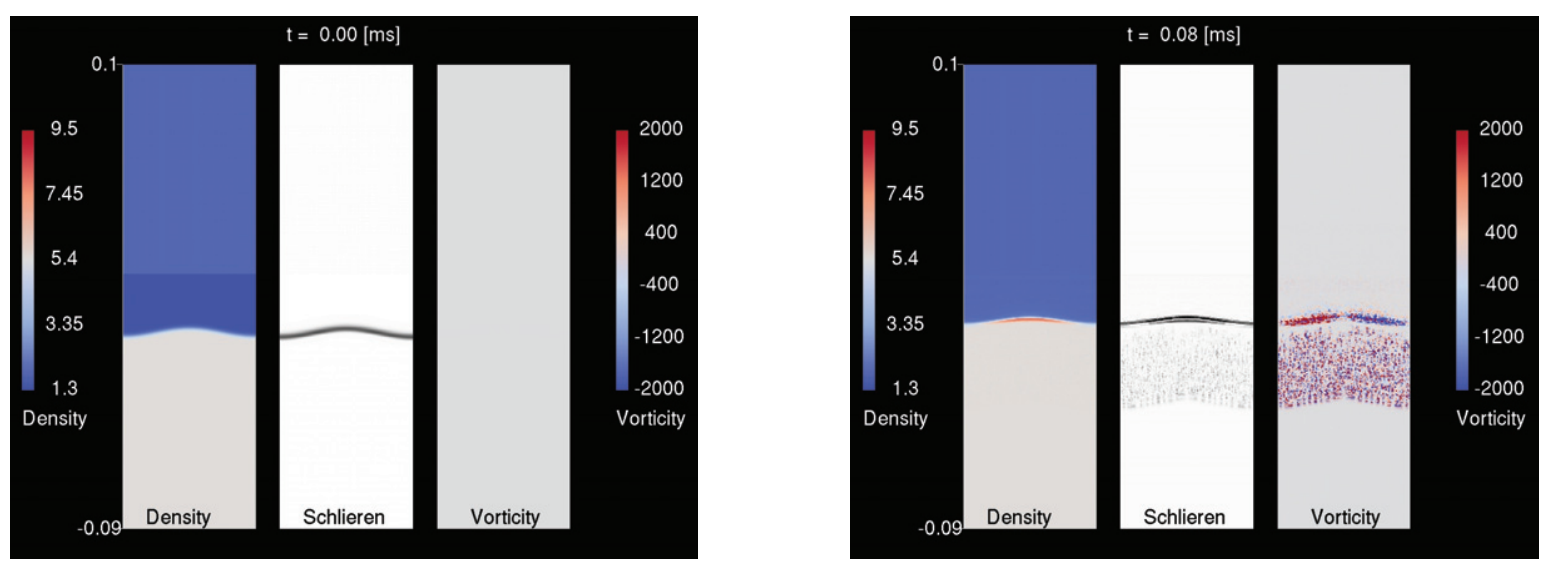

(b) $t=0.08 \mathrm{~ms}$.

Figure 7: Density, simulated Schlieren, and vorticity $(|\nabla \times \mathbf{u}|)$ for the single-mode RichtmyerMeshkov instability $(P=2,128$ cells per wavelength). Conservative transport equation and limiting of the conserved variables (fully conservative approach A).

consistent fashion using an exponential diffusion function [67], with a diffusion length of $0.5 \mathrm{~cm}$. We impose periodic boundaries on the sides and zero-gradient conditions at the entrance and exit of the shock tube. The gases have an initial upward velocity such that the interface remains in the domain after the shock interaction.

With the fully conservative approach A, pressure oscillations are produced and lead to negative densities early in time, causing the simulations to fail just after the shock interaction (Fig. 7). Even with approach B, pressure errors at the interface are significant. These errors in pressure modify the velocity field, and therefore the vorticity, to eventually distort the interface morphology (Fig. 8). Furthermore, these initially small errors lead to a loss in symmetry at late times, as explained in [19].

These oscillations are avoided when using our approach $\mathrm{C}$ as evidenced by comparing the vorticity contours of Fig. 9 to those of Fig. 8. The early-time growth of the instability (before reshock) from the simulation agrees well with the experimental data, as shown through convergence of the perturbation amplitude in the integral sense on a sequence of grids (Fig. 10). With our approach, we can accurately simulate the physics of such problems, unlike certain prior studies that had to assume gases of constant $\gamma$ [67].

\subsection{Interaction of a Shock in Water with a Gas Bubble}

We consider a shock initiated in water impinging upon a cylindrical (two-dimensional) air bubble to assess our approach for more complicated equations of state, and for high pressures and large density ratios. An air bubble of radius $r_{0}=1$ initially lies at rest in water at atmospheric pressure; surface tension is neglected. A Mach 1.82 shock, corresponding to a pressure ratio of $1.9 \times 10^{4}$, is initialized in the water upstream of the bubble at $x=-2[68,28,69,70]$. The density, velocity, and pressure, non-dimensionalized by the properties of air at atmospheric pressure, in the different 


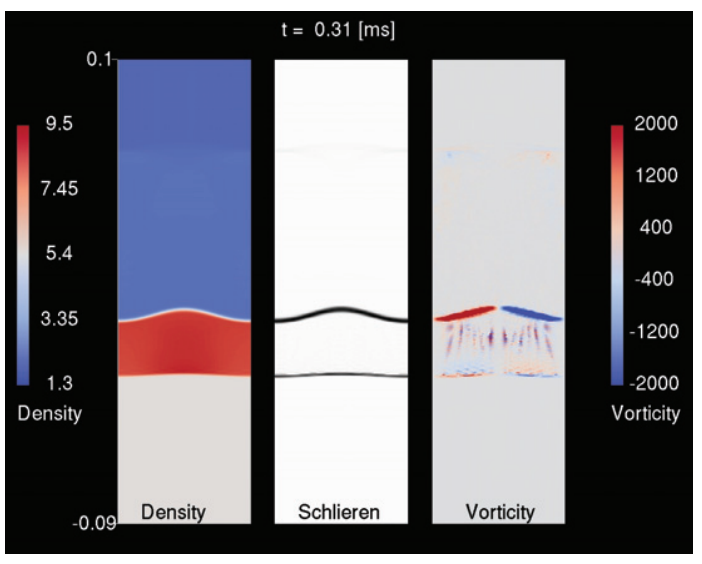

(a) $t=0.31 \mathrm{~ms}$

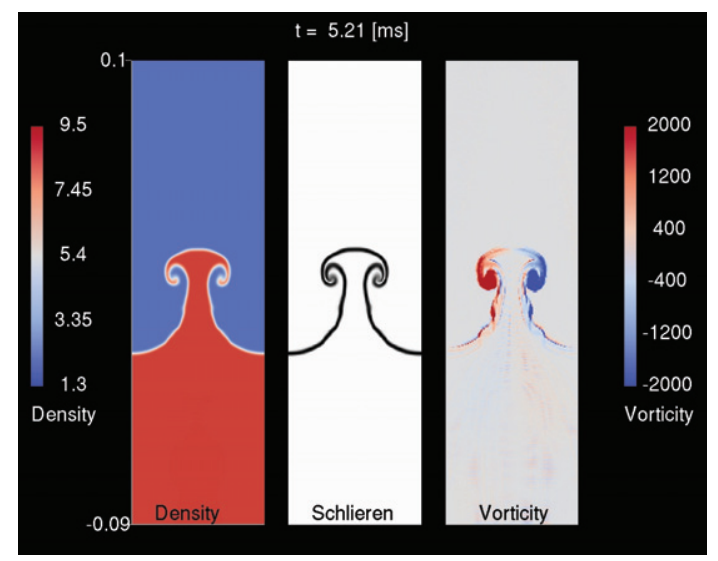

(b) $t=5.21 \mathrm{~ms}$

Figure 8: Density, simulated Schlieren, and vorticity for the single-mode Richtmyer-Meshkov instability $(P=2,128$ cells per wavelength) using non-conservative transport equation and limiting of the conserved variables (approach B).

parts of the domain are

$$
(\rho, u, p)= \begin{cases}(1,0,0.71), & \text { in the bubble } \\ (846.58,0,0.71), & \text { in the water upstream of the shock } \\ \left(1078.12,644.28,1.3610^{5}\right), & \text { in the water downstream of the shock }\end{cases}
$$

For air, $\gamma=1.4$ and $B=0$ bars. The values of these properties for water for the stiffened equation of state are determined through empirical fits [71, 72]: $\gamma=5.5$ and $B=4921.15$ bars [73].

Fig. 11 shows a sequence of simulated Schlieren contours for this problem using our approach C. As in past simulations a re-entrant jet forms and impacts the opposite side of the bubble, thereby generating a strong shock moving radially outward. When limiting the conserved variables (approaches A and B), large spurious pressure oscillations are generated, which lead to negative densities as the shock starts to interact with the bubble; thus, the simulations fail. For such large pressure and density ratios, higher resolution (e.g., order of accuracy, grid refinement or Riemann solver) may lead to large negative pressures in the numerically diffuse interface regions and thus cause simulations to fail for reasons beyond the scope of this article $[74,75,14,21]$. For this reason, we use the Rusanov flux solver for this problem only.

\section{Conclusions}

In this article, we introduc a solution-adaptive Discontinuous Galerkin method to simulate compressible multiphase flows with shocks and interfaces in a stable and accurate fashion. Following the interface-capturing approach of Abgrall [1], we model flows of multiple fluid components or phases using a single equation of state with varying material properties. To represent compressible phenomena in solids, liquids and gases, we consider the Mie-Grüneisen family of equations of state, which describes a wide range of media, including stiffened and ideal gas equations of state. We 


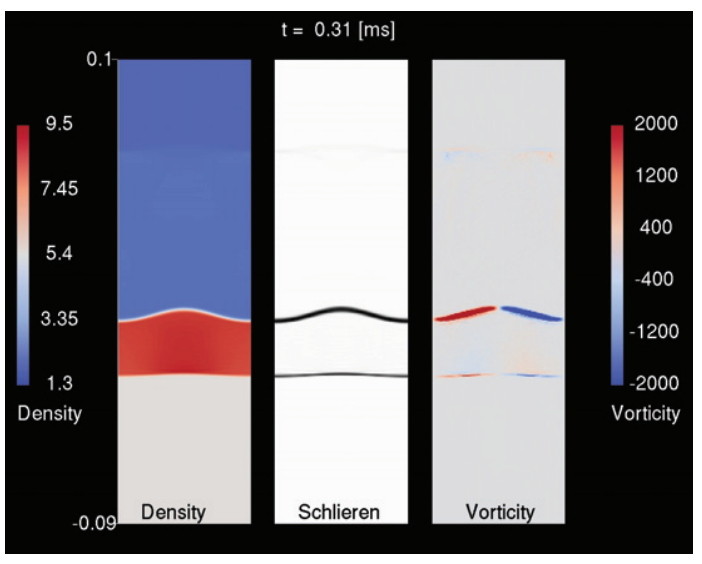

(a) $t=0.31 \mathrm{~ms}$.

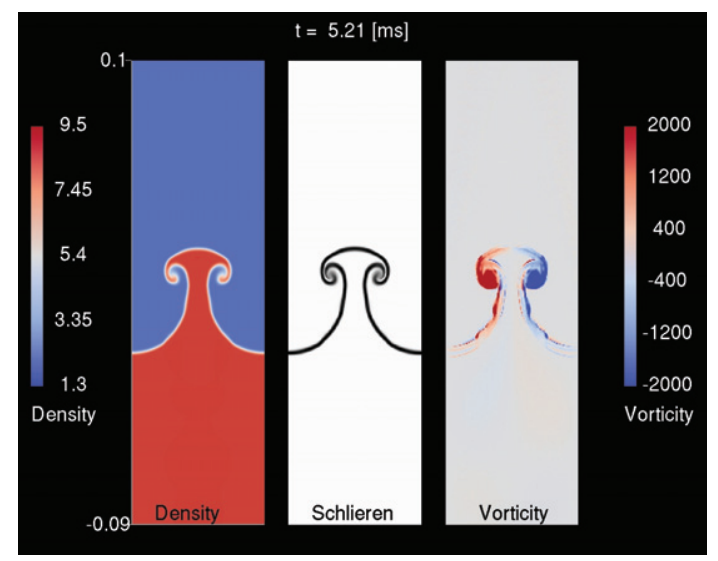

(b) $t=5.21 \mathrm{~ms}$.

Figure 9: Density, simulated Schlieren, and vorticity for the single-mode Richtmyer-Meshkov instability using our approach ( $P=2,128$ cells per wavelength).

show why spurious pressure oscillations occur in the DG framework when material properties vary, and how to remedy this problem. Using stringent test problems in one and two dimensions, we verify our approach. We can make the following conclusions:

- We develop a DG method that is conservative, non-oscillatory at interfaces (and shocks) and high-order accurate in smooth regions.

- Two key steps must be followed to avoid spurious pressure oscillations at interfaces between fluids of different material properties:

1. Transport equations for appropriate material properties must be solved in a suitable weak form. E.g., for ideal gases, a non-conservative equation for $1 /(\gamma-1)$ must be solved, as suggested by Abgrall [1] for FV methods.

2. Solution limiting must be applied to the appropriate variables (density, momentum, pressure, and the appropriate properties in the equation of state) to result in a nonoscillatory, conservative, and high-order accurate procedure. Limiting of the primitive variables does not ensure conservation. Our proposed approach does not require more operations than fully conservative limiting.

- We develop a new characteristic-based discontinuity sensor inspired by the physics for shocks, interfaces, and contacts.

- We can handle strong shocks in multi-dimensional settings involving possibly large density ratios for gas/gas, gas/liquid and fluid/solid interfaces in which the media obey an equation of state in the Mie-Grüneisen family.

- Results indicate that the largest errors are produced when solving the transport equation in conservative form and limiting the conserved variables (approach A). This approach fails for relatively simple problems involving gas/gas interfaces. Using a non-conservative transport 


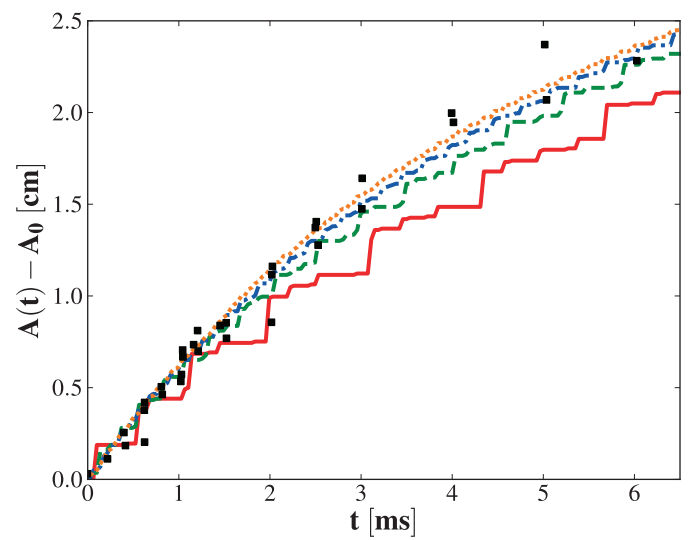

Figure 10: Amplitude growth vs. $t$ for the single-mode Richtmyer-Meshkov instability using our approach $\mathrm{C}(P=2)$. Black square: experimental data [66]. Solid red: 16 cells per wavelength; dashed green: 32; dash-dot blue: 64; dotted orange: 128 .

equation and limiting the conserved variables (approach B) produces non-negligeable errors shown to affect interface morphology in gas/gas problems and produce negative pressures in gas/liquid problems.

This work sets the basis for subsequent multiphysics research with DG using GPUs. We are also exploring potential inconsistencies with temperature fields for the compressible Navier-Stokes equations [76].

\section{Acknowledgements}

The authors gratefully acknowledge discussions with Pooya Movahed, Sreenivas Varadan, and Rui Fang. This research was supported in part by the DOE NNSA/ASC under the predictive Science Academic Alliance Program by Grant No. DEFC52-08NA28616, by ONR grant N00014-121-0751 under Dr. Ki-Han Kim, by NSF grant CBET 1253157, and through computational resources and services provided by Advanced Research Computing at the University of Michigan, Ann Arbor.

\section{Appendix A. Proofs of the Nonlinear Properties of Limiting}

Appendix A.1. Addition

Let $U=A+B$, with two different limiting approaches $\widetilde{U}=\widetilde{A+B}$ and $\widetilde{\widetilde{U}}=\widetilde{A}+\widetilde{B}$. We denote three adjacent computational cells $L: x \in[-3,-1], C: x \in[-1,1]$, and $R: x \in[1,3]$. Without loss of generality, we assume for simplicity $P=1$ and HR limiting. In this case,

$$
A(x)=A_{0}+A_{1} x, \quad \widetilde{A}(x)=A_{0}+\widetilde{A}_{1} x, \quad B(x)=B_{0}+B_{1} x, \quad \widetilde{B}(x)=B_{0}+\widetilde{B}_{1} x .
$$

where $\widetilde{A}_{1}=\frac{1}{2} \operatorname{minmod}\left(A_{0}^{C}-A_{0}^{L}, A_{0}^{R}-A_{0}^{C}\right)$ and $\widetilde{B}_{1}=\frac{1}{2} \operatorname{minmod}\left(B_{0}^{C}-B_{0}^{L}, B_{0}^{R}-B_{0}^{C}\right)$. We can write the limited slopes of $U$ as

$\widetilde{U}_{1}=\frac{1}{2} \operatorname{minmod}\left(U_{0}^{C}-U_{0}^{L}, U_{0}^{R}-U_{0}^{C}\right)=\frac{1}{2} \operatorname{minmod}\left(\left(A_{0}^{C}-A_{0}^{L}\right)+\left(B_{0}^{C}-B_{0}^{L}\right),\left(A_{0}^{R}-A_{0}^{C}\right)-\left(A_{0}^{R}-A_{0}^{C}\right)\right)$, 


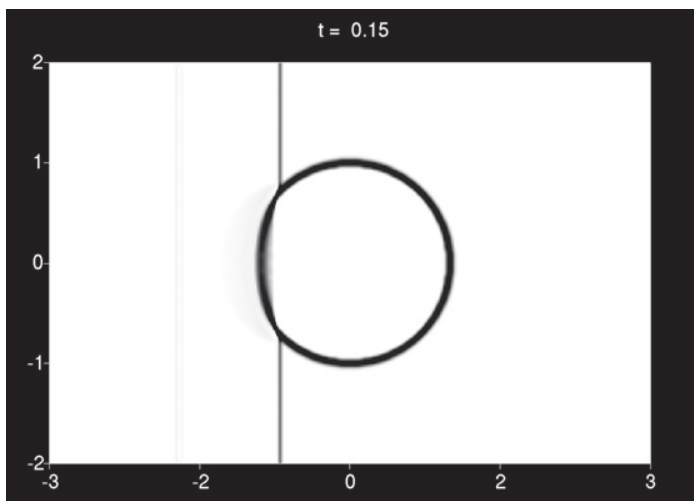

(a) $t=0.15$.

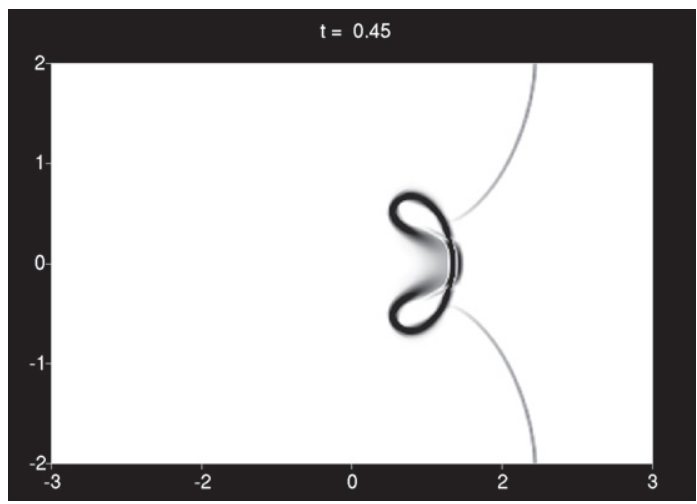

(c) $t=0.45$.

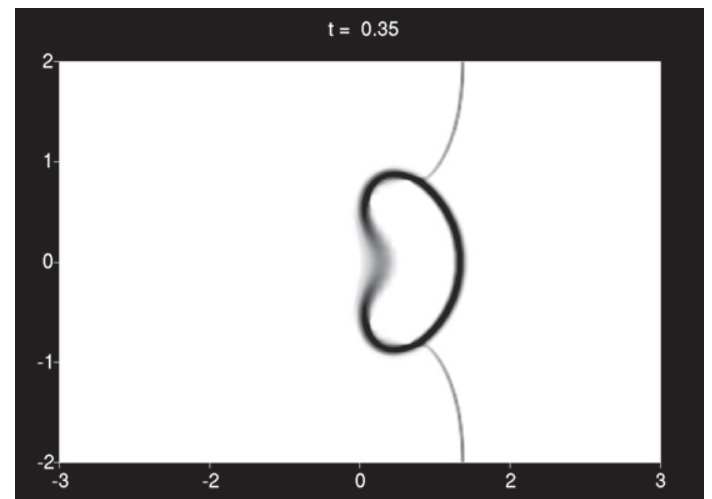

(b) $t=0.35$.

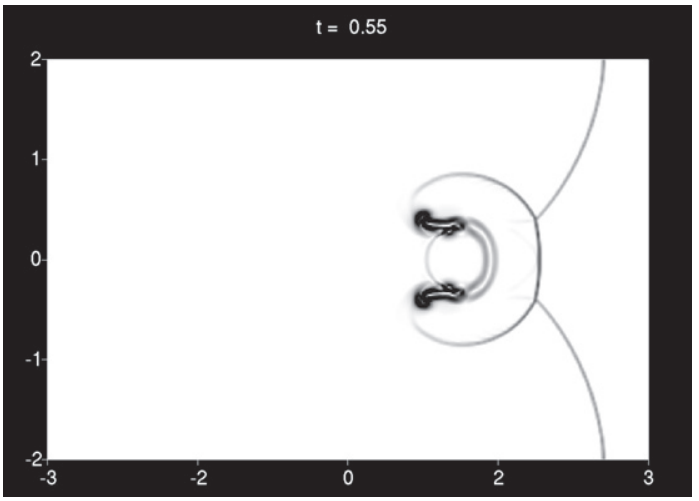

(d) $t=0.55$

Figure 11: Simulated Schlieren of density for a strong shock impacting a two-dimensional air bubble in water using our approach $\mathrm{C}(P=2,128$ cells per diameter $)$.

and, in the other case,

$$
\widetilde{\widetilde{U}}_{1}=\widetilde{A}_{1}+\widetilde{B}_{1}=\frac{1}{2}\left(\operatorname{minmod}\left(A_{0}^{C}-A_{0}^{L}, A_{0}^{R}-A_{0}^{C}\right)+\operatorname{minmod}\left(B_{0}^{C}-B_{0}^{L}, B_{0}^{R}-B_{0}^{C}\right)\right) .
$$

The nonlinearity of the minmod procedure implies that $\widetilde{U}_{1} \neq \widetilde{\widetilde{U}}_{1}$ and, therefore, $\widetilde{A+B} \neq \widetilde{A}+\widetilde{B}$.

\section{Appendix A.2. Multiplication}

Let $U=A B$ and $\widetilde{U}=\widetilde{A} \widetilde{B}$. Without loss of generality, we assume $P=2$ and HR limiting. Through polynomial identification,

$$
\widetilde{U}_{0}=\widetilde{A}_{0} \widetilde{B}_{0}, \quad \widetilde{U}_{1}=\widetilde{A}_{1} \widetilde{B}_{0}+\widetilde{A}_{0} \widetilde{B}_{1}, \quad \widetilde{U}_{2}=\widetilde{A}_{2} \widetilde{B}_{0}+\widetilde{A}_{1} \widetilde{B}_{1}+\widetilde{A}_{0} \widetilde{B}_{2} .
$$

The cell averages of $U$ and $\widetilde{U}$ are

$$
\begin{aligned}
& \int U \mathrm{~d} \Omega=U_{0}+\frac{1}{6} U_{2}=A_{0} B_{0}+\frac{1}{6}\left(A_{2} B_{0}+A_{1} B_{1}+A_{0} B_{2}\right), \\
& \int \widetilde{U} \mathrm{~d} \Omega=\widetilde{U}_{0}+\frac{1}{6} \widetilde{U}_{2}=\widetilde{A}_{0} \widetilde{B}_{0}+\frac{1}{6}\left(\widetilde{A}_{2} \widetilde{B}_{0}+\widetilde{A}_{1} \widetilde{B}_{1}+\widetilde{A}_{0} \widetilde{B}_{2}\right) .
\end{aligned}
$$


We can compute the difference in the cell averages of $U$ and $\widetilde{U}$ to obtain

$$
\int \widetilde{U} \mathrm{~d} \Omega-\int U \mathrm{~d} \Omega=A_{0} B_{0}+\frac{1}{6}\left(\widetilde{A}_{1} \widetilde{B}_{1}-A_{1} B_{1}\right)-\frac{1}{6^{2}}\left(\widetilde{A}_{2} \widetilde{B}_{2}-A_{2} B_{2}\right) \neq 0 .
$$

Since this difference is non-zero in general, this implies that $\int \widetilde{A} \widetilde{B} \mathrm{~d} \Omega \neq \int A B \mathrm{~d} \Omega$.

\section{Appendix B. Extension to Other Multiphase Models}

We illustrate the generality of our limiting approach by applying it to the five-equations model, also used to describe multiphase flows [36], written for a two-phase system as:

$$
\begin{aligned}
& \frac{\partial \alpha_{1}}{\partial t}+u_{j} \frac{\partial \alpha_{1}}{\partial x_{j}}=0, \\
& \frac{\partial\left(\rho_{1} \alpha_{1}\right)}{\partial t}+\frac{\partial}{\partial x_{j}}\left(\rho_{1} \alpha_{1} u_{j}\right)=0, \\
& \frac{\partial\left(\rho_{2} \alpha_{2}\right)}{\partial t}+\frac{\partial}{\partial x_{j}}\left(\rho_{2} \alpha_{2} u_{j}\right)=0, \\
& \frac{\partial\left(\rho u_{i}\right)}{\partial t}+\frac{\partial}{\partial x_{j}}\left(\rho u_{i} u_{j}+p \delta_{i j}\right)=0, \\
& \frac{\partial E}{\partial t}+\frac{\partial}{\partial x_{j}}\left[u_{j}(E+p)\right]=0,
\end{aligned}
$$

where $\rho_{i}$ and $\alpha_{i}$ are the density and volume fraction of the $i^{\text {th }}$ fluid, respectively, $\alpha_{2}=1-\alpha_{1}$ and $\rho=\alpha_{1} \rho_{1}+\alpha_{2} \rho_{2}$. The mixture internal energy is defined as $\rho e=\alpha_{1} \rho_{1} e_{1}+\alpha_{2} \rho_{2} e_{2}$. Gryngarten and Menon [35] discuss a primitive reconstruction procedure but do not address possible conservation issues arising from limiting the primitive variables. For high-order accurate, non-oscillatory, and conservative limiting, we directly apply our limiting procedure detailled above. For a Mie-Grüneisen equation of state, we limit $p$ and $\alpha_{1}$ and reconstruct the internal energy as

$$
\begin{aligned}
\widetilde{\rho e}_{n} & =\frac{1}{\Gamma_{1}} \sum_{k=0}^{n}\left(\begin{array}{l}
n \\
k
\end{array}\right) \widetilde{p}_{n-k} \widetilde{\left(\alpha_{1}\right)_{k}}+\left(-\frac{p_{\text {ref }, 1}}{\Gamma_{1}}+\rho e_{\text {ref }, 1}\right) \widetilde{\left(\alpha_{1}\right)_{n}} \\
& +\frac{1}{\Gamma_{2}} \sum_{k=0}^{n}\left(\begin{array}{l}
n \\
k
\end{array}\right) \widetilde{p}_{n-k} \widetilde{\left(\alpha_{2}\right)_{k}}+\left(-\frac{p_{\text {ref }, 2}}{\Gamma_{2}}+\rho e_{\text {ref }, 2}\right) \widetilde{\left(\alpha_{2}\right)_{n}}, \quad \text { for } n=1, \ldots, P,
\end{aligned}
$$

where $\widetilde{\alpha_{2}}=1-\widetilde{\alpha_{1}}$. Our limiting procedure can readily be extended to other models, e.g., BaerNunziato [30], in this way.

[1] R. Abgrall, How to prevent pressure oscillations in multicomponent flow calculations: A quasi conservative approach, J. Comput. Phys. 125 (1996) 150-160.

[2] S. Adjerid, K. D. Devine, J. E. Flaherty, L. Krivodonova, A posteriori error estimation for discontinuous Galerkin solutions of hyperbolic problems, Comput. Methods Appl. Mech. Engrg. 191 (2002) 1097-1112.

[3] S. Adjerid, T. C. Massey, Superconvergence of discontinuous Galerkin solutions for a nonlinear scalar hyperbolic problem, Comput. Methods Appl. Mech. Engrg. 195 (2006) 3331-3346. 
[4] B. Cockburn, G. Lin, C.-W. Shu, TVB Runge-Kutta local projection discontinuous Galerkin finite element method for conservation laws III: One-dimensional systems, J. Comput. Phys. 84 (1989) 90-113.

[5] B. Cockburn, C.-W. Shu, TVB Runge-Kutta local projection discontinuous Galerkin finite element method for conservation laws II: General framework, Math. Comput. 52 (1989) 411435 .

[6] B. Cockburn, Hou, C.-W. Shu, The Runge-Kutta local projection discontinuous Galerkin finite element method for conservation laws IV: The multidimensional case, Math. Comput. $54(1990) 545-581$.

[7] B. Cockburn, C.-W. Shu, The local discontinuous Galerkin method for time-dependent convection-diffusion systems, SIAM J. Numer. Anal. 35 (1997) 2440-2463.

[8] B. Cockburn, C.-W. Shu, The Runge-Kutta discontinuous Galerkin method for conservation laws V: Multidimensional systems, J. Comput. Phys. 141 (1997) 199-224.

[9] R. Biswas, K. D. Devine, J. E. Flaherty, Parallel, adaptive finite element methods for conservation laws, Appl. Numer. Math. 14 (1994) 255-283.

[10] L. Krivodonova, Limiters for high-order discontinuous Galerkin methods, J. Comput. Phys. 226 (2007) 879-896.

[11] D. Kuzmin, A vertex-based hierarchical slope limiter for p-adaptive discontinuous Galerkin methods, J. Comput. Appl. Math. 233 (2010) 3077-3085.

[12] Y. Liu, C.-W. Shu, E. Tadmor, M. Zhang, Central discontinuous Galerkin methods on overlapping cells with a nonoscillatory hierarchical reconstruction, SIAM J. Numer. Anal. 45 (2007) 2442 .

[13] Z. Xu, Y. Liu, C.-W. Shu, Hierarchical reconstruction for discontinuous Galerkin methods on unstructured grids with a WENO-type linear reconstruction and partial neighboring cells, J. Comput. Phys. 228 (2009) 2194-2212.

[14] R. Saurel, R. Abgrall, A simple method for compressible multifluid flows, SIAM J. Sci. Comput. 21 (1999) 1115-1145.

[15] R. Abgrall, S. Karni, Computations of compressible multifluids, J. Comput. Phys. 169 (2001) 594-623.

[16] E. Johnsen, T. Colonius, Implementation of WENO schemes in compressible multicomponent flow problems, J. Comput. Phys. 219 (2006) 715-732.

[17] T. Xiong, C.-W. Shu, M. Zhang, WENO Scheme with Subcell Resolution for Computing Nonconservative Euler Equations with Applications to One-Dimensional Compressible TwoMedium Flows, J. Sci. Comput. 53 (2012) 222-247.

[18] S. Kawai, H. Terashima, A high-resolution scheme for compressible multicomponent flows with shock waves, Int. J. Numer. Methods Fluids 66 (2011) 1207-1225. 
[19] P. Movahed, E. Johnsen, A solution-adaptive method for efficient compressible multifluid simulations, with application to the Richtmyer-Meshkov instability, J. Comput. Phys. 239 (2013) 166-186.

[20] K.-M. Shyue, An efficient shock-capturing algorithm for compressible multicomponent problems, J. Comput. Phys. 142 (1998) 208-242.

[21] K.-M. Shyue, A Fluid-Mixture Type Algorithm for Compressible Multicomponent Flow with van der Waals Equation of State, J. Comput. Phys. 156 (1999) 43-88.

[22] K.-M. Shyue, A Fluid-Mixture Type Algorithm for Compressible Multicomponent Flow with Mie-Grüneisen Equation of State, J. Comput. Phys. 171 (2001) 678-707.

[23] E. Johnsen, T. Colonius, Numerical simulations of non-spherical bubble collapse, J. Fluid Mech. 629 (2009) 231-262.

[24] G. Ward, D. I. Pullin, A hybrid, center-difference, limiter method for simulations of compressible multicomponent flows with Mie-Grüneisen equation of state, J. Comput. Phys. 229 (2010) 2999-3018.

[25] R. Saurel, R. Abgrall, A multiphase Godunov method for compressible multifluid and multiphase flows, J. Comput. Phys. 150 (1999) 425-467.

[26] R. Abgrall, R. Saurel, Discrete equations for physical and numerical compressible multiphase mixtures, J. Comput. Phys. 186 (2003) 361-396.

[27] G. Murrone, H. Guillard, A five equation reduced model for compressible two phase flow problems, J. Comput. Phys. 202 (2005) 664-698.

[28] R. K. Shukla, C. Pantano, J. B. Freund, An interface capturing method for the simulation of multi-phase compressible flows, J. Comput. Phys. 229 (2010) 7411-7439.

[29] S. Tokareva, E. Toro, HLLC-type Riemann solver for the Baer-Nunziato equations of compressible two-phase flow, J. Comput. Phys. 229 (2010) 3573-3604.

[30] E. Franquet, V. Perrier, Runge-Kutta discontinuous Galerkin method for the approximation of Baer and Nunziato type multiphase models, J. Comput. Phys. 231 (2012) 4096-4141.

[31] C. Michoski, J. Evans, P. Schmitz, A. Vasseur, A discontinuous Galerkin method for viscous compressible multifluids, J. Comput. Phys. 229 (2010) 2249-2266.

[32] B. Cockburn, C.-w. Shu, Runge - Kutta Discontinuous Galerkin Methods for ConvectionDominated Problems 16 (2001).

[33] E. Franquet, V. Perrier, Runge-Kutta discontinuous Galerkin method for interface flows with a maximum preserving limiter, Comput. Fluids 65 (2012) 2-7.

[34] C. Wang, C.-W. Shu, An interface treating technique for compressible multi-medium flow with Runge-Kutta discontinuous Galerkin method, J. Comput. Phys. 229 (2010) 8823-8843. 
[35] L. D. Gryngarten, S. Menon, A generalized approach for sub- and super-critical flows using the Local Discontinuous Galerkin method, Comput. Methods Appl. Mech. Eng. 253 (2013) $169-185$.

[36] G. Allaire, S. Clerc, S. Kokh, A Five-Equation Model for the Simulation of Interfaces between Compressible Fluids, J. Comput. Phys. 181 (2002) 577-616.

[37] R. Menikoff, B. J. Plohr, The Riemann problem for fluid flow of real materials, Rev. Mode. Phys. 61 (1989) 75-130.

[38] A. B. Wardlaw, Jr., H. U. Mair, Spherical solutions of an underwater explosion bubble, Shock Vib. 5 (1979) 89-.

[39] G. Cochran, J. Chan, Shock initiation and detonation models in one and two dimensions, UCID 18024 (LLNL, 1979).

[40] P. G. LeFloch, Shock waves for nonlinear hyperbolic systems in nonconservative form, Institute for Math. and its Appl., Minneapolis, preprint 593 (1989) 1989.

[41] G. Dal Maso, P. G. LeFloch, F. Murat, Definition and weak stability of nonconservative products, J. Math. Pures Appl. 74 (1995) 483-548.

[42] C. Parés, Numerical methods for nonconservative hyperbolic systems: A theoretical framework, SIAM J. Numer. Anal. 44 (2006) 300.

[43] M. J. Castro, P. G. LeFloch, M. L. Muñoz Ruiz, C. Parés, Why many theories of shock waves are necessary: Convergence error in formally path-consistent schemes, J. Comput. Phys. 227 (2008) 8107-8129.

[44] A. Canestrelli, A. Siviglia, M. Dumbser, E. F. Toro, Well-balanced high-order centred schemes for non-conservative hyperbolic systems. Applications to shallow water equations with fixed and mobile bed, Adv. Water Resour. 32 (2009) 834-844.

[45] S. Rhebergen, O. Bokhove, J. van der Vegt, Discontinuous Galerkin finite element methods for hyperbolic nonconservative partial differential equations, J. Comput. Phys. 227 (2008) $1887-1922$.

[46] W. Sollie, O. Bokhove, J. van der Vegt, Space-time discontinuous Galerkin finite element method for two-fluid flows, J. Comput. Phys. 230 (2011) 789-817.

[47] A. Harten, P. D. Lax, B. van Leer, On upstream differencing and Godunov-type schemes for hyperbolic conservation laws, SIAM review 25 (1983) 35-61.

[48] P. D. Lax, Weak solutions of nonlinear hyperbolic equations and their numerical computation, Commun. Pure Appl. Math. 7 (1954) 159-193.

[49] V. Rusanov, The calculation of the interaction of non-stationary shock waves and obstacles, Comput. Math. Math. Phys. 1 (1962) 304-320.

[50] E. F. Toro, M. Spruce, W. Speares, Restoration of the contact surface in the HLL-Riemann solver, Shock Waves 4 (1994) 25-34. 
[51] M. Dumbser, E. F. Toro, A Simple Extension of the Osher Riemann Solver to Non-conservative Hyperbolic Systems, J. Sci. Comput. 48 (2010) 70-88.

[52] I. Toumi, A weak formulation of Roe's approximate Riemann solver, J. Comput. Phys. 102 (1992) 360-373.

[53] P. L. Roe, Approximate Riemann solvers, parameter vectors, and difference schemes, J. Comput. Phys. 43 (1981) 357-372.

[54] B. van Leer, Towards the ultimate conservative difference scheme. V. A second-order sequel to Godunov's method, J. Comput. Phys. 32 (1979) 101-136.

[55] X.-D. Liu, S. Osher, T. Chan, Weighted Essentially Non-oscillatory Schemes, J. Comput. Phys. 115 (1994) 200-212.

[56] E. Johnsen, J. Larsson, A. V. Bhagatwala, W. H. Cabot, P. Moin, B. J. Olson, P. S. Rawat, S. K. Shankar, B. Sjogreen, H. C. Yee, X. Zhong, S. K. Lele, Assessment of high resolution methods for numerical simulations of compressible turbulence, J. Comput. Phys. 228 (2010) $1213-1237$.

[57] Y.-X. Ren, M. Liu, H. Zhang, A characteristic-wise hybrid compact-WENO scheme for solving hyperbolic conservation laws, J. Comput. Phys. 192 (2003) 365-386.

[58] M. Lombardini, Richtmyer-Meshkov instability in converging geometries, Ph.D. thesis, California Institute of Technology, 2008.

[59] C. Geuzaine, J.-F. Remacle, Gmsh: A 3-D finite element mesh generator with built-in preand post-processing facilities, Int. J. Numer. Methods Eng. 79 (2009) 1309-1331.

[60] M. T. Henry de Frahan, E. Johnsen, Numerical simulations of a shock interacting with successive interfaces using the Discontinuous Galerkin method: the multilayered Richtmyer-Meshkov and Rayleigh-Taylor instabilities, Submitted. (2014).

[61] C. A. Di Stefano, G. Malamud, M. T. Henry de Frahan, C. C. Kuranz, A. Shimony, S. R. Klein, R. P. Drake, E. Johnsen, D. Shvarts, V. A. Smalyuk, D. Martinez, Observation and modeling of mixing-layer development in high-energy-density, blast-wave-driven shear flow, Phys. Plasmas 21 (2014) 056306.

[62] C. Shu, S. Osher, Efficient implementation of essentially non-oscillatory shock-capturing schemes II, J. Comput. Phys. 83 (1989) 32-78.

[63] T. Liu, B. Khoo, K. Yeo, Ghost fluid method for strong shock impacting on material interface, J. Comput. Phys. 190 (2003) 651-681.

[64] E. Johnsen, Analysis of Numerical Errors Generated by Slowly Moving Shock Waves, AIAA J. 51 (2013) 1269-1274.

[65] S. Jin, J.-G. Liu, The Effects of Numerical Viscosities, J. Comput. Phys. 126 (1996) 373-389.

[66] B. D. Collins, J. W. Jacobs, PLIF flow visualization and measurements of the RichtmyerMeshkov instability of an air/SF6 interface, J. Fluid Mech. 464 (2002) 113-136. 
[67] M. Latini, O. Schilling, W. S. Don, Effects of WENO flux reconstruction order and spatial resolution on reshocked two-dimensional Richtmyer-Meshkov instability, J. Comput. Phys. 221 (2007) 805-836.

[68] G. Ball, B. Howell, T. Leighton, M. Schofield, Shock-induced collapse of a cylindrical air cavity in water: a Free-Lagrange simulation, Shock Waves 10 (2000) 265-276.

[69] X. Hu, B. Khoo, N. Adams, F. Huang, A conservative interface method for compressible flows, J. Comput. Phys. 219 (2006) 553-578.

[70] R. Nourgaliev, T. Theofanous, High-fidelity interface tracking in compressible flows: Unlimited anchored adaptive level set, J. Comput. Phys. 224 (2007) 836-866.

[71] S. P. Marsh, LASL Shock Hugoniot Data, Univ. California Press, Berkeley, CA, 1980.

[72] K. S. Holian, T-4 Handbook of Material Properties Data Bases, Technical Report LA-10160MS, Los Alamos National Laboratory, Los Alamos, NM, 1984.

[73] J. P. Cocchi, R. Saurel, J. C. Loraud, Treatment of interface problems with Godunov-type schemes, Shock Waves 5 (1996) 347-357.

[74] B. Einfeldt, C. Munz, P. Roe, B. Sjögreen, On Godunov-type methods near low densities, J. Comput. Phys. 92 (1991) 273-295.

[75] J. J. Quirk, A contribution to the great Riemann solver debate, Int. J. Numer. Methods Fluids 18 (1994) 555-574.

[76] E. Johnsen, F. Ham, Preventing numerical errors generated by interface-capturing schemes in compressible multi-material flows, J. Comput. Phys. 231 (2012) 5705-5717. 